NISTIR 8399

\title{
Interlaboratory Results: Candidate Aqueous Film Forming Foam (AFFF) Reference Materials
}

Jessica L. Reiner

Benjamin J. Place

This publication is available free of charge from:

https://doi.org/10.6028/NIST.IR.8399

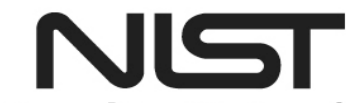

National Institute of Standards and Technology U.S. Department of Commerce 


\title{
Interlaboratory Results: Candidate Aqueous Film Forming Foam (AFFF) Reference Materials
}

\author{
Jessica L. Reiner \\ Benjamin J. Place \\ Chemical Sciences Division \\ Material Measurement Laboratory
}

This publication is available free of charge from:

https://doi.org/10.6028/NIST.IR.8399

October 2021

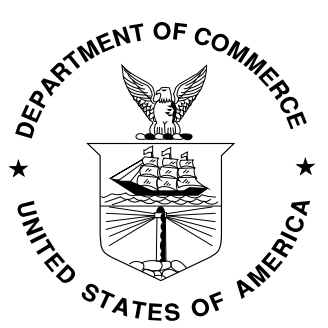

U.S. Department of Commerce

Gina M. Raimondo, Secretary

National Institute of Standards and Technology James K. Olthoff, Performing the Non-Exclusive Functions and Duties of the Under Secretary of Commerce for Standards and Technology \& Director, National Institute of Standards and Technology 
Certain commercial entities, equipment, or materials may be identified in this document in order to describe an experimental procedure or concept adequately. Such identification is not intended to imply recommendation or endorsement by the National Institute of Standards and Technology, nor is it intended to imply that the entities, materials, or equipment are necessarily the best available for the purpose.

National Institute of Standards and Technology Interagency or Internal Report 8399

Natl. Inst. Stand. Technol. Interag. Intern. Rep. 8399, 57 pages (October 2021)

This publication is available free of charge from: https://doi.org/10.6028/NIST.IR.8399 


\section{Introduction}

The measurement of per- and polyfluorinated substances (PFAS) in complex environmental matrices has been proven to have significant interlaboratory variability that can affect the confidence of quantitative measurements. ${ }^{1-3}$ In addition to high variability in measurements, currently there are no EPA published methods for analysis of PFAS in media other than drinking water. Without published methods there is the potential for greater variability in analytical results from laboratory to laboratory. To this end, there have been multiple statements of needs from Department of Defense (DoD) agencies to focus on validation, accuracy, and reproducibility of PFAS data in a variety of matrices. The National Institute of Standards and Technology has provided measurements of PFAS on ten different reference materials, including human serum, human plasma, fish tissue, house dust, soil, and domestic sludge. These materials are useful for the development and validation of methods; however, the concentrations in these reference materials are significantly lower than the concentrations in source materials impacting contaminated DoD sites, such as aqueous film forming foam (AFFF). A SERDP (Strategic Environmental Research and Development Program) project was funded, starting in FY19, to develop an AFFF PFAS reference material (Project code ER18-1664). The goal is to provide a material to help evaluate the ability of laboratories to analyze high concentrations of PFAS in AFFF concentrates.

This summary report documents and discusses the results from the PFAS AFFF interlaboratory exercise. Twelve laboratories responded to the call for participants. Samples were shipped to participants in January 2020 and results were returned to NIST by December 31, 2020.

\section{Materials and Data Treatment}

\section{Materials and Sample Preparation}

Four different AFFF formulations were shipped to NIST Gaithersburg between March and April of 2019. Fisherbrand cryogenic storage vials ( $2 \mathrm{~mL}$ capacity), lot number 1244025 , and were used to bottle the AFFFs. Fifty vials of each AFFF were created. Volumetrically, approximately $1 \mathrm{~mL}$ of each AFFF was added to the storage vials. The four materials were labeled as candidate Reference Materials (RMs) 8690 Per- and Polyfluoroalkyl Substances (PFAS) in Aqueous FilmForming Foams (AFFF) Formulation I, 8691 Per- and Polyfluoroalkyl Substances (PFAS) in Aqueous Film-Forming Foams (AFFF) Formulation II, 8692 Per- and Polyfluoroalkyl Substances (PFAS) in Aqueous Film-Forming Foams (AFFF) Formulation III, and 8693 Per- and Polyfluoroalkyl Substances (PFAS) in Aqueous Film-Forming Foams (AFFF) Formulation IV. The homogeneity of these materials was assessed by examining PFAS in a stratified random sampling of the materials $(n=5)$ before they were shipped out to participants. Samples were shipped to participants in January 2020 and results were returned to NIST by December 31, 2020.

Thirty-three PFAS were examined in the four candidate RMs. Participants were not required to measure every analyte specified in the study (Table 1) but were asked to provide data for the analytes they could measure. Participants were asked to use their in-house analytical methods to determine the mass fraction of the different PFAS in $\mathrm{mg} / \mathrm{kg}$ in each of the materials. All values reported by the laboratories are totals, inclusive of the linear and branched isomers. 
Table 1. Analytes (measurands) and their acronyms

\begin{tabular}{|c|c|}
\hline Measurand $^{\mathrm{a}}$ & Acronym \\
\hline Perfluorobutanoic acid & PFBA \\
\hline Perfluoropentanoic acid & PFPeA \\
\hline Perfluorohexanoic acid & PFHxA \\
\hline Perfluoroheptanoic acid & PFHpA \\
\hline Perfluorooctanoic acid & PFOA \\
\hline Perfluorononanoic acid & PFNA \\
\hline Perfluorodecanoic acid & PFDA \\
\hline Perfluoroundecanoic acid & PFUnA \\
\hline Perfluorododecanoic acid & PFDoA \\
\hline Perfluorotridecanoic acid & PFTrA \\
\hline Perfluorotetradecanoic acid & PFTA \\
\hline Perfluorobutanesulfonic acid & PFBS \\
\hline Perfluoropentanesulfonic acid & PFPeS \\
\hline Perfluorohexanesulfonic acid & PFHxS \\
\hline Perfluoroheptanesulfonic acid & PFHpS \\
\hline Perfluorooctanesulfonic acid & PFOS \\
\hline Perfluorononanesulfonic acid & PFNS \\
\hline Perfluorodecanesulfonic acid & PFDS \\
\hline Perfluorododecanesulfonic acid & PFDoS \\
\hline Perfluorooctanesulfonamide & FOSA \\
\hline N-Methyl perfluorooctanesulfonamide & NMeFOSA \\
\hline N-Ethyl perfluorooctanesulfonamide & NEtFOSA \\
\hline N-Methyl perfluorooctanesulfonamidoacetic acid & NMeFOSAA \\
\hline N-Ethyl perfluorooctanesulfonamidoacetic acid & NEtFOSAA \\
\hline N-Methyl perfluorooctanesulfonamidoethanol & NMeFOSE \\
\hline N-Ethyl perfluorooctanesulfonamidoethanol & NEtFOSE \\
\hline $1 \mathrm{H}, 1 \mathrm{H}, 2 \mathrm{H}, 2 \mathrm{H}$-Perfluorohexane sulfonic acid & $4: 2$ FTS \\
\hline $1 \mathrm{H}, 1 \mathrm{H}, 2 \mathrm{H}, 2 \mathrm{H}-\mathrm{Perfluorooctane} \mathrm{sulfonic}$ acid & $6: 2$ FTS \\
\hline $1 \mathrm{H}, 1 \mathrm{H}, 2 \mathrm{H}, 2 \mathrm{H}$-Perfluorodecane sulfonic acid & $8: 2$ FTS \\
\hline 4,8-dioxa-3H-perfluorononanoic acid & ADONA \\
\hline Hexafluoropropylene oxide dimer acid & HFPO-DA \\
\hline 9-Chlorohexadecafluoro-3-oxanonane-1-sulfonic acid & 9Cl-PF3ONS \\
\hline 11-Chloroeicosafluoro-3-oxaundecane-1-sulfonic acid & 11Cl-PF3OUnDS \\
\hline
\end{tabular}

${ }^{a}$ Measurands are totals, inclusive of linear and branched isomers 


\section{Data Treatment}

Community tables and figures are provided using randomized laboratory codes, with identities only know to NIST and the individual laboratories. The statistical approaches are outlined below for each type of data representation.

\section{$\underline{\text { Statistics }}$}

Data tables and graphs throughout this report contain information about the performance of each laboratory relative to that of the other participants in this study. All calculations were performed in PROLab Plus (QuoData GmbH, Dresden, Germany). The consensus means and standard deviation are calculated according to the robust Q/Hampel method outlined in ISO 13528:2015(E), Annex C. ${ }^{4}$ In the Q/Hampel method, the standard deviation is estimated by means of the Q method, based on the consideration of pairwise absolute difference. The standard deviation is then used for the Hampel estimation of the mean, which is based on the principle of limiting extreme values in the data.

\section{Summary Data Tables}

These data tables include a summary of all reported data for a particular analyte (measurand) in a particular AFFF candidate RM. Participants can compare the data for their laboratory to data reported by the other participating laboratories or to the consensus data. Consensus means and standard deviations are calculated using the laboratory means. ${ }^{1}$

\section{Graphs}

Individual laboratory data (diamonds) are plotted with the individual laboratory standard deviation (rectangles). Laboratories reporting values as "below LOQ" can still be successful in the study if the target value is also below the laboratory LOQ. The black solid line represents the consensus mean, and the green shaded area represents the $95 \%$ confidence interval for the consensus mean, based on the standard error of the consensus mean. The uncertainty in the consensus mean is calculated using the equation below, based on the repeatability standard deviation $\left(s_{\mathrm{r}}\right)$, the reproducibility standard deviation $\left(S_{\mathrm{R}}\right)$, the number of participants reporting data, and the average number of replicates reported by each participant. The uncertainty about the consensus mean is independent of the range of tolerance.

$u_{\text {mean }}=\sqrt{\frac{s_{R}^{2}-s_{r}^{2}}{n_{\text {particpants }}}+\frac{s_{R}^{2}}{n_{\text {participants }} \times n_{\text {Average Number of Replicates per Participant }}}}$

The solid red lines represent the range of tolerance (values that result in an acceptable $Z$ ' score, $\left|Z^{\prime}\right| \leq 2$ ). If the lower limit is below zero, the lower limit has been set to zero. In this view, the relative locations of individual laboratory data and consensus zones with respect to the target zone can be compared easily. 


\section{Candidate RM 8690 Per- and Polyfluoroalkyl Substances (PFAS) in Aqueous Film-Forming Foams (AFFF) Formulation I}

For candidate RM 8690 a summary of the data report by the laboratories and the community results is provided in Table 2 . The individual analytes are discussed in subsequent sections along with figures.

Table 2. Reported mass fraction of PFAS (mean \pm standard deviation in $\mathrm{mg} / \mathrm{kg}$ as received) in candidate RM 8690 from participating laboratories

\begin{tabular}{|c|c|c|c|c|c|c|c|c|}
\hline & \multicolumn{6}{|c|}{ Individual Results } & \multicolumn{2}{|c|}{ Community Results } \\
\hline & $\begin{array}{l}\text { LC0003 } \\
(n=3)\end{array}$ & $\begin{array}{l}\text { LC0004 } \\
(n=3)\end{array}$ & $\begin{array}{l}\text { LC0006 } \\
(n=3)\end{array}$ & $\begin{array}{l}\text { LC0007 } \\
\qquad(n=3)\end{array}$ & $\begin{array}{l}\text { LC0011 } \\
\qquad(n=3)\end{array}$ & $\begin{array}{l}\text { LC0013 } \\
(n=3)\end{array}$ & $\begin{array}{c}\text { Consensus } \\
\text { Mean }\end{array}$ & $\begin{array}{c}\text { Consensus } \\
\text { Standard } \\
\text { Deviation }\end{array}$ \\
\hline PFBA & $0.707 \pm 0.145$ & $1.25 \pm 0.04$ & $1.33 \pm 0.07$ & $1.37 \pm 0.50$ & $1.11 \pm 0.01$ & $1.20 \pm 0.00$ & 1.18 & 0.22 \\
\hline PFPeA & $1.20 \pm 0.33$ & $1.37 \pm 0.03$ & $1.84 \pm 0.06$ & $1.40 \pm 0.05$ & $1.15 \pm 0.03$ & $1.33 \pm 0.01$ & 1.33 & 0.16 \\
\hline PFHxA & $2.44 \pm 0.57$ & $3.11 \pm 0.16$ & $3.81 \pm 0.28$ & $3.06 \pm 0.46$ & $2.96 \pm 0.08$ & $2.93 \pm 0.06$ & 3.02 & 0.38 \\
\hline PFHрA & $0.487 \pm 0.146$ & $0.830 \pm 0.053$ & $1.52 \pm 0.07$ & $0.457 \pm 0.010$ & $0.928 \pm 0.017$ & $0.859 \pm 0.017$ & 0.808 & 0.318 \\
\hline PFOA & $2.06 \pm 0.48$ & $2.90 \pm 0.04$ & $4.30 \pm 0.44$ & $3.74 \pm 0.62$ & $3.01 \pm 0.12$ & $2.51 \pm 0.19$ & 3.09 & 0.90 \\
\hline PFNA & NR & $<0.470$ & NR & $0.02 \pm 0.00$ & $0.01 \pm 0.00$ & NR & 0.014 & 0.003 \\
\hline PFDA & $0.190 \pm 0.000$ & $<0.470$ & NR & $<0.013$ & $0.01 \pm 0.00$ & NR & 0.098 & 0.306 \\
\hline PFUnA & $0.130 \pm 0.000$ & $<0.470$ & NR & $<0.013$ & NR & NR & & \\
\hline PFDoA & NR & $<0.470$ & NR & $<0.050$ & NR & NR & & \\
\hline PFTrA & NR & $<0.470$ & NR & $<0.050$ & NR & NR & & \\
\hline PFTA & NR & $<0.470$ & NR & $<0.250$ & NR & NR & & \\
\hline PFBS & $4.18 \pm 0.92$ & $5.25 \pm 0.23$ & $5.58 \pm 0.52$ & $5.42 \pm 0.05$ & $4.81 \pm 0.22$ & $5.14 \pm 0.16$ & 5.06 & 0.59 \\
\hline PFPeS & $6.26 \pm 1.37$ & $3.60 \pm 0.08$ & $7.34 \pm 0.36$ & $4.01 \pm 0.08$ & $4.22 \pm 0.13$ & $3.83 \pm 0.18$ & 4.45 & 0.84 \\
\hline PFHxS & $21.8 \pm 5.0$ & $22.1 \pm 0.6$ & $35.1 \pm 2.9$ & $31.7 \pm 1.4$ & $27.2 \pm 0.9$ & $23.7 \pm 0.6$ & 26.9 & 7.0 \\
\hline PFHpS & $0.657 \pm 0.045$ & $2.73 \pm 0.07$ & $8.54 \pm 1.02$ & $4.22 \pm 0.13$ & $3.28 \pm 0.16$ & $2.51 \pm 0.07$ & 3.38 & 2.35 \\
\hline PFOS & $147 \pm 27$ & $138 \pm 5$ & $157 \pm 10$ & $200 \pm 2$ & $165 \pm 2$ & $185 \pm 4$ & 165 & 39 \\
\hline PFNS & NR & $<0.470$ & NR & $0.075 \pm 0.004$ & $0.163 \pm 0.012$ & NR & 0.119 & 0.178 \\
\hline PFDS & $0.070 \pm 0.026$ & $<0.470$ & NR & $0.171 \pm 0.006$ & $0.195 \pm 0.003$ & NR & 0.145 & 0.064 \\
\hline PFDoS & NR & $<0.470$ & NR & NR & $0.025 \pm 0.004$ & NR & & \\
\hline FOSA & NR & $<0.470$ & NR & $<0.013$ & $0.010 \pm 0.001$ & NR & 0.013 & 0.009 \\
\hline NMeFOSA & NR & NR & NR & $<0.050$ & $0.001 \pm 0.000$ & NR & & \\
\hline NEtFOSA & NR & NR & NR & $<0.050$ & NR & NR & & \\
\hline NMeFOSAA & NR & $<0.470$ & NR & $0.113 \pm 0.016$ & NR & NR & & \\
\hline NEtFOSAA & NR & $<0.470$ & NR & $<0.025$ & NR & NR & & \\
\hline NMeFOSE & NR & NR & NR & $4.12 \pm 0.58$ & NR & NR & & \\
\hline NEtFOSE & NR & NR & NR & $<0.250$ & NR & NR & & \\
\hline 4:2 FTS & NR & $<0.470$ & NR & $<0.012$ & $0.011 \pm 0.001$ & NR & & \\
\hline $6: 2$ FTS & $0.430 \pm 0.030$ & $1.92 \pm 0.19$ & $2.31 \pm 0.36$ & $1.16 \pm 0.04$ & $1.69 \pm 0.06$ & $3.42 \pm 1.56$ & 1.82 & 1.18 \\
\hline 8:2 FTS & $4.02 \pm 1.19$ & $<0.470$ & NR & $0.222 \pm 0.005$ & $0.313 \pm 0.004$ & NR & 0.268 & 0.214 \\
\hline ADONA & NR & $<0.470$ & NR & $0.151 \pm 0.031$ & NR & NR & & \\
\hline HFPO-DA & NR & $<0.470$ & NR & $3.82 \pm 0.20$ & NR & NR & & \\
\hline 9Cl-PF3ONS & NR & $<0.470$ & NR & NR & NR & NR & & \\
\hline 11Cl-PF3OUnDS & NR & $<0.470$ & NR & NR & NR & $\mathrm{NR}$ & & \\
\hline
\end{tabular}

Values shown as "<" a specified number indicate the actual reporting limit provided by the laboratory

NR: Not Reported 


\section{Candidate RM 8691 Per- and Polyfluoroalkyl Substances (PFAS) in Aqueous Film-Forming Foams (AFFF) Formulation II}

For candidate RM 8691 a summary of the data report by the laboratories and the community results is provided in Table 3 . The individual analytes are discussed in subsequent sections along with figures.

Table 3. Reported mass fraction of PFAS (mean \pm standard deviation in $\mathrm{mg} / \mathrm{kg}$ as received) in candidate RM 8691 from participating laboratories

\begin{tabular}{|c|c|c|c|c|c|c|c|c|}
\hline & \multicolumn{6}{|c|}{ Individual Results } & \multicolumn{2}{|c|}{ Community Results } \\
\hline & $\begin{array}{l}\text { LC0003 } \\
(n=3)\end{array}$ & $\begin{array}{l}\text { LC0004 } \\
(n=3)\end{array}$ & $\begin{array}{l}\text { LC0006 } \\
(n=3)\end{array}$ & $\begin{array}{l}\text { LC0007 } \\
(n=3)\end{array}$ & $\begin{array}{l}\text { LC0011 } \\
\qquad(n=3)\end{array}$ & $\begin{array}{c}\text { LC0013 } \\
(n=3)\end{array}$ & $\begin{array}{c}\text { Consensus } \\
\text { Mean }\end{array}$ & $\begin{array}{c}\text { Consensus } \\
\text { Standard } \\
\text { Deviation }\end{array}$ \\
\hline PFBA & $0.067 \pm 0.006$ & $<0.470$ & NR & $<1.00$ & $0.071 \pm 0.002$ & $0.066 \pm 0.001$ & 0.068 & 0.006 \\
\hline PFPeA & $0.353 \pm 0.012$ & $<0.470$ & $0.247 \pm 0.020$ & $0.094 \pm 0.006$ & $0.065 \pm 0.001$ & $0.062 \pm 0.003$ & 0.146 & 0.078 \\
\hline PFHxA & $0.137 \pm 0.015$ & $<0.470$ & $0.223 \pm 0.004$ & $0.195 \pm 0.014$ & $0.157 \pm 0.002$ & $0.166 \pm 0.006$ & 0.175 & 0.042 \\
\hline PFHрA & $0.030 \pm 0.000$ & $<0.470$ & NR & $0.021 \pm 0.001$ & $0.009 \pm 0.000$ & $0.055 \pm 0.008$ & 0.029 & 0.024 \\
\hline PFOA & $0.027 \pm 0.006$ & $<0.470$ & NR & $<0.096$ & $0.037 \pm 0.002$ & $0.045 \pm 0.002$ & 0.036 & 0.015 \\
\hline PFNA & NR & $<0.470$ & NR & $<0.013$ & $0.003 \pm 0.000$ & $0.017 \pm 0.002$ & 0.014 & 0.004 \\
\hline PFDA & $0.197 \pm 0.006$ & $<0.470$ & NR & $0.024 \pm 0.002$ & $0.021 \pm 0.000$ & $0.020 \pm 0.002$ & 0.022 & 0.007 \\
\hline PFUnA & $0.130 \pm 0.000$ & $<0.470$ & NR & $0.014 \pm 0.001$ & $0.001 \pm 0.000$ & NR & 0.011 & 0.028 \\
\hline PFDoA & NR & $<0.470$ & NR & $<0.050$ & $0.009 \pm 0.001$ & NR & & \\
\hline PFTrA & NR & $<0.470$ & NR & $<0.050$ & $0.001 \pm 0.000$ & NR & & \\
\hline PFTA & NR & $<0.470$ & $0.029 \pm 0.009$ & $<0.050$ & $0.005 \pm 0.001$ & NR & 0.017 & 0.04 \\
\hline PFBS & NR & $<0.470$ & $0.347 \pm 0.026$ & $0.761 \pm 0.665$ & NR & NR & 0.554 & 0.686 \\
\hline PFPeS & NR & $<0.470$ & NR & $0.029 \pm 0.004$ & NR & NR & & \\
\hline PFHxS & NR & $<0.470$ & NR & $0.054 \pm 0.014$ & NR & NR & & \\
\hline PFHpS & NR & $<0.470$ & $0.391 \pm 0.105$ & $<0.012$ & NR & NR & & \\
\hline PFOS & NR & $<0.470$ & $1.43 \pm 1.06$ & $<0.023$ & NR & NR & & \\
\hline PFNS & NR & $<0.470$ & NR & $<0.024$ & NR & NR & & \\
\hline PFDS & NR & $<0.470$ & NR & $<0.012$ & NR & NR & & \\
\hline PFDoS & NR & $<0.470$ & NR & NR & NR & NR & & \\
\hline FOSA & NR & $<0.470$ & NR & $<0.013$ & NR & NR & & \\
\hline NMeFOSA & NR & NR & NR & $<0.050$ & NR & NR & & \\
\hline NEtFOSA & NR & NR & NR & $<0.050$ & NR & NR & & \\
\hline NMeFOSAA & NR & $<0.470$ & NR & $<0.100$ & NR & NR & & \\
\hline NEtFOSAA & NR & $<0.470$ & NR & $<0.025$ & NR & NR & & \\
\hline NMeFOSE & NR & NR & NR & $<0.050$ & NR & NR & & \\
\hline NEtFOSE & NR & NR & NR & $<0.050$ & NR & NR & & \\
\hline 4:2 FTS & NR & $<0.470$ & NR & $0.017 \pm 0.003$ & $0.001 \pm 0.000$ & NR & 0.009 & 0.031 \\
\hline $6: 2$ FTS & $0.630 \pm 0.061$ & $0.563 \pm 0.059$ & NR & $0.629 \pm 0.073$ & $0.697 \pm 0.019$ & $2.02 \pm 0.26$ & 0.630 & 0.154 \\
\hline $8: 2$ FTS & NR & $<0.470$ & NR & $0.037 \pm 0.006$ & $0.056 \pm 0.006$ & $0.472 \pm 0.129$ & 0.047 & 0.053 \\
\hline ADONA & NR & $<0.470$ & NR & $<0.047$ & NR & NR & & \\
\hline HFPO-DA & NR & $<0.470$ & NR & $<0.997$ & NR & NR & & \\
\hline 9Cl-PF3ONS & NR & $<0.470$ & NR & NR & NR & NR & & \\
\hline 11Cl-PF3OUnDS & NR & $<0.470$ & NR & NR & NR & NR & & \\
\hline
\end{tabular}

Values shown as " $<$ " a specified number indicate the actual reporting limit provided by the laboratory

NR: Not Reported 


\section{Candidate RM 8692 Per- and Polyfluoroalkyl Substances (PFAS) in Aqueous Film-Forming Foams (AFFF) Formulation III}

For candidate RM 8692 a summary of the data report by the laboratories and the community results is provided in Table 4 . The individual analytes are discussed in subsequent sections along with figures.

Table 4. Reported mass fraction of PFAS (mean \pm standard deviation in $\mathrm{mg} / \mathrm{kg}$ as received) in candidate RM 8692 from participating laboratories

\begin{tabular}{|c|c|c|c|c|c|c|c|c|}
\hline & \multicolumn{6}{|c|}{ Individual Results } & \multicolumn{2}{|c|}{ Community Results } \\
\hline & $\begin{array}{l}\text { LC0003 } \\
(n=3)\end{array}$ & $\begin{array}{l}\text { LC0004 } \\
(n=3)\end{array}$ & $\begin{array}{c}\text { LC0006 } \\
(n=3)\end{array}$ & $\begin{array}{c}\text { LC0007 } \\
(n=3)\end{array}$ & $\begin{array}{l}\text { LC0011 } \\
\qquad(n=3)\end{array}$ & $\begin{array}{l}\text { LC0013 } \\
(n=3)\end{array}$ & $\begin{array}{l}\text { Consensus } \\
\text { Mean }\end{array}$ & $\begin{array}{c}\text { Consensus } \\
\text { Standard } \\
\text { Deviation }\end{array}$ \\
\hline PFBA & $0.127 \pm 0.023$ & $<0.470$ & $\mathrm{NR}$ & $<1.000$ & $0.156 \pm 0.001$ & $0.146 \pm 0.003$ & 0.143 & 0.017 \\
\hline PFPeA & NR & $<0.470$ & $0.193 \pm 0.011$ & $0.034 \pm 0.004$ & $0.011 \pm 0.000$ & NR & 0.066 & 0.058 \\
\hline PFHxA & $0.073 \pm 0.012$ & $<0.470$ & NR & $0.103 \pm 0.007$ & $0.091 \pm 0.003$ & $0.101 \pm 0.006$ & 0.092 & 0.019 \\
\hline PFHpA & NR & $<0.470$ & $2.32 \pm 0.09$ & $<0.050$ & $0.002 \pm 0.000$ & $0.038 \pm 0.004$ & 0.020 & 0.085 \\
\hline PFOA & NR & $<0.470$ & NR & $<0.096$ & NR & NR & & \\
\hline PFNA & NR & $<0.470$ & NR & $<0.013$ & NR & NR & & \\
\hline PFDA & NR & $<0.470$ & NR & $0.037 \pm 0.009$ & NR & NR & & \\
\hline PFUnA & $0.130 \pm 0.000$ & $<0.470$ & NR & $<0.013$ & NR & NR & & \\
\hline PFDoA & NR & $<0.470$ & NR & $<0.025$ & NR & NR & & \\
\hline PFTrA & NR & $<0.470$ & NR & $<0.050$ & NR & NR & & \\
\hline PFTA & NR & $<0.470$ & NR & $<0.050$ & NR & NR & & \\
\hline PFBS & NR & $<0.470$ & $0.404 \pm 0.037$ & $0.049 \pm 0.008$ & NR & NR & 0.227 & 0.718 \\
\hline PFPeS & NR & $<0.470$ & NR & $0.023 \pm 0.007$ & NR & NR & & \\
\hline PFHxS & NR & $<0.470$ & NR & $0.021 \pm 0.005$ & NR & NR & & \\
\hline PFHpS & NR & $<0.470$ & NR & $<0.012$ & NR & NR & & \\
\hline PFOS & NR & $<0.470$ & $4.14 \pm 0.19$ & $0.020 \pm 0.002$ & NR & NR & 2.08 & 8.75 \\
\hline PFNS & NR & $<0.470$ & $0.144 \pm 0.030$ & $<0.024$ & NR & NR & & \\
\hline PFDS & NR & $<0.470$ & NR & $<0.012$ & NR & NR & & \\
\hline PFDoS & NR & $<0.470$ & NR & NR & NR & NR & & \\
\hline FOSA & NR & $<0.470$ & NR & $<0.013$ & NR & NR & & \\
\hline NMeFOSA & NR & NR & NR & $<0.050$ & NR & NR & & \\
\hline NEtFOSA & NR & NR & NR & $<0.050$ & NR & NR & & \\
\hline NMeFOSAA & NR & $<0.470$ & NR & $<0.100$ & NR & NR & & \\
\hline NEtFOSAA & NR & $<0.470$ & NR & $<0.025$ & NR & NR & & \\
\hline NMeFOSE & NR & NR & NR & $<0.050$ & NR & NR & & \\
\hline NEtFOSE & NR & NR & NR & $<0.050$ & NR & NR & & \\
\hline 4:2 FTS & NR & $<0.470$ & NR & $0.019 \pm 0.003$ & NR & NR & & \\
\hline $6: 2$ FTS & $0.357 \pm 0.067$ & $0.573 \pm 0.025$ & NR & $0.292 \pm 0.011$ & $0.503 \pm 0.006$ & $1.28 \pm 0.09$ & 0.527 & 0.256 \\
\hline $8: 2$ FTS & NR & $<0.470$ & NR & $<0.012$ & NR & $0.228 \pm 0.114$ & & \\
\hline ADONA & NR & $<0.470$ & NR & $<0.047$ & NR & NR & & \\
\hline HFPO-DA & NR & $<0.470$ & NR & $<0.997$ & NR & NR & & \\
\hline 9Cl-PF3ONS & NR & $<0.470$ & NR & NR & NR & NR & & \\
\hline 11Cl-PF3OUnDS & NR & $<0.470$ & NR & NR & NR & NR & & \\
\hline
\end{tabular}

Values shown as "<" a specified number indicate the actual reporting limit provided by the laboratory

NR: Not Reported 


\section{Candidate RM 8693 Per- and Polyfluoroalkyl Substances (PFAS) in Aqueous Film-Forming Foams (AFFF) Formulation IV}

For candidate RM 8693 a summary of the data report by the laboratories and the community results is provided in Table 5. The individual analytes are discussed in subsequent sections along with figures.

Table 5. Reported mass fraction of PFAS (mean \pm standard deviation in $\mathrm{mg} / \mathrm{kg}$ as received) in candidate RM 8693 from participating laboratories

\begin{tabular}{|c|c|c|c|c|c|c|c|c|}
\hline & \multicolumn{6}{|c|}{ Individual Results } & \multicolumn{2}{|c|}{ Community Results } \\
\hline & $\begin{array}{l}\mathrm{LC} 0003 \\
(n=3)\end{array}$ & $\begin{array}{c}\text { LC0004 } \\
\quad(n=3)\end{array}$ & $\begin{array}{l}\text { LC0006 } \\
(n=3)\end{array}$ & $\begin{array}{c}\text { LC0007 } \\
(n=3)\end{array}$ & $\begin{array}{l}\text { LC0011 } \\
(n=3)\end{array}$ & $\begin{array}{l}\text { LC0013 } \\
(n=3)\end{array}$ & $\begin{array}{c}\text { Consensus } \\
\text { Mean }\end{array}$ & $\begin{array}{c}\text { Consensus } \\
\text { Standard } \\
\text { Deviation }\end{array}$ \\
\hline$\overline{\text { PFBA }}$ & $0.347 \pm 0.296$ & $0.547 \pm 0.015$ & $0.990 \pm 0.018$ & $<1.000$ & $0.462 \pm 0.012$ & $0.497 \pm 0.045$ & 0.528 & 0.174 \\
\hline PFPeA & $0.443 \pm 0.038$ & $<0.470$ & $0.586 \pm 0.033$ & $0.183 \pm 0.016$ & $0.142 \pm 0.003$ & $0.161 \pm 0.017$ & 0.260 & 0.104 \\
\hline PFHxA & $1.46 \pm 0.30$ & $1.94 \pm 0.04$ & $3.40 \pm 0.14$ & $1.70 \pm 0.12$ & $1.60 \pm 0.04$ & $1.65 \pm 0.19$ & 1.72 & 0.58 \\
\hline PFHpA & $0.037 \pm 0.006$ & $<0.470$ & $4.12 \pm 0.24$ & $<0.050$ & $0.021 \pm 0.001$ & $0.057 \pm 0.010$ & 0.041 & 0.041 \\
\hline PFOA & NR & $<0.470$ & NR & $<0.096$ & NR & NR & & \\
\hline PFNA & NR & $<0.470$ & NR & $<0.013$ & NR & NR & & \\
\hline PFDA & NR & $<0.470$ & NR & $<0.013$ & NR & NR & & \\
\hline PFUnA & NR & $<0.470$ & NR & $<0.013$ & NR & NR & & \\
\hline PFDoA & NR & $<0.470$ & NR & $<0.050$ & NR & NR & & \\
\hline PFTrA & NR & $<0.470$ & NR & $<0.050$ & NR & NR & & \\
\hline PFTA & NR & $<0.470$ & $0.030 \pm 0.001$ & $<0.050$ & NR & NR & & \\
\hline PFBS & NR & $<0.470$ & $0.547 \pm 0.015$ & $<0.013$ & NR & NR & 0.282 & 1.155 \\
\hline PFPeS & NR & $<0.470$ & NR & $<0.012$ & NR & NR & & \\
\hline PFHxS & NR & $<0.470$ & NR & $<0.013$ & NR & NR & & \\
\hline PFHpS & NR & $<0.470$ & NR & $0.049 \pm 0.002$ & NR & NR & & \\
\hline PFOS & NR & $<0.470$ & $4.33 \pm 0.25$ & $<0.012$ & NR & NR & & \\
\hline PFNS & NR & $<0.470$ & NR & $<0.012$ & NR & NR & & \\
\hline PFDS & NR & $<0.470$ & NR & $<0.012$ & NR & NR & & \\
\hline PFDoS & NR & $<0.470$ & NR & NR & NR & NR & & \\
\hline FOSA & NR & $<0.470$ & NR & $<0.013$ & NR & NR & & \\
\hline NMeFOSA & NR & NR & NR & $<0.050$ & NR & NR & & \\
\hline NEtFOSA & NR & NR & NR & $<0.050$ & NR & NR & & \\
\hline NMeFOSAA & NR & $<0.470$ & NR & $<0.100$ & NR & NR & & \\
\hline NEtFOSAA & NR & $<0.470$ & NR & $<0.025$ & NR & NR & & \\
\hline NMeFOSE & NR & NR & NR & $0.139 \pm 0.008$ & NR & NR & & \\
\hline NEtFOSE & NR & NR & NR & $<0.050$ & NR & NR & & \\
\hline $4: 2$ FTS & $0.300 \pm 0.066$ & $<0.470$ & $0.733 \pm 0.030$ & $0.324 \pm 0.008$ & $0.323 \pm 0.026$ & $0.372 \pm 0.052$ & 0.33 & 0.08 \\
\hline 6:2 FTS & $37.5 \pm 4.0$ & $128 \pm 2$ & $168 \pm 7$ & $130 \pm 2$ & $152 \pm 0.371$ & $137 \pm 10$ & 133 & 34 \\
\hline $8: 2$ FTS & NR & NR & $250 \pm 9$ & $0.013 \pm 0.001$ & $0.030 \pm 0.001$ & $0.293 \pm 0.017$ & 0.112 & 0.579 \\
\hline ADONA & NR & $<0.470$ & NR & $<0.047$ & NR & NR & & \\
\hline HFPO-DA & NR & $<0.470$ & NR & $<0.997$ & NR & NR & & \\
\hline 9Cl-PF3ONS & NR & $<0.470$ & NR & NR & NR & NR & & \\
\hline 11Cl-PF3OUnDS & NR & $<0.470$ & NR & NR & NR & NR & & \\
\hline
\end{tabular}

Values shown as "<" a specified number indicate the actual reporting limit provided by the laboratory

NR: Not Reported 


\section{Individual Analytes}

\section{Perfluorobutanoic acid (PFBA)}

- $\quad$ Six laboratories reported results for PFBA in candidate RMs 8690 and 8693, while five laboratories reported results for PFBA in candidate RMs 8691 and 8692

- $\quad$ The between-laboratory variability was good for candidate RMs 8690,8691 , and $8692(19 \%, 9 \%$, and $12 \%$ relative standard deviation (RSD), respectively)

- $\quad$ The between-laboratory variability was poor for candidate RM 8693 (33 \% RSD)

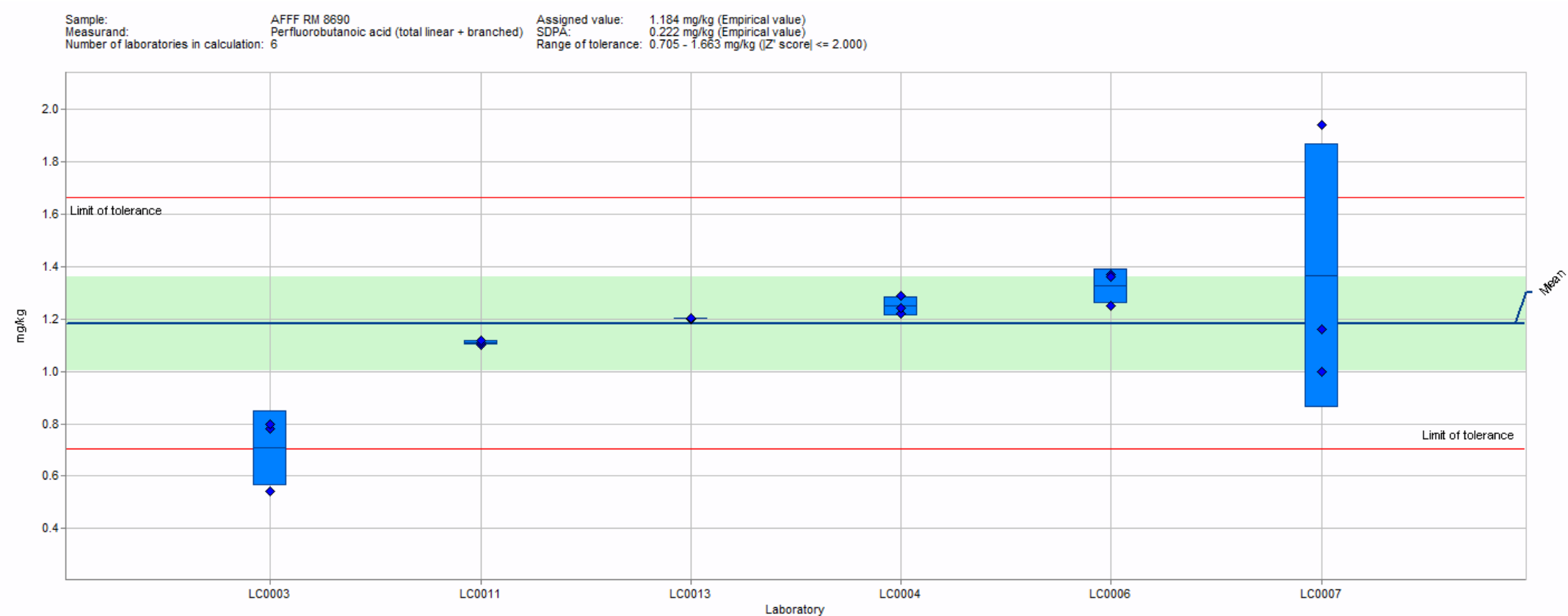

Figure 1-1. PFBA in candidate RM 8690 Per- and Polyfluoroalkyl Substances (PFAS) in Aqueous Film-Forming Foams (AFFF) Formulation I. In this view, individual laboratory data are plotted (diamonds) with the individual laboratory standard deviation (rectangle). The solid blue line represents the consensus mean, and the green shaded region represents the $95 \%$ confidence interval for the consensus mean. The red solid lines represent the consensus range of tolerance, calculated as the values above and below the consensus mean that result in an acceptable $\mathrm{Z}_{\text {comm }}^{\prime}$ score, $\left|\mathrm{Z}_{\text {comm }}^{\prime}\right| \leq 2$. 


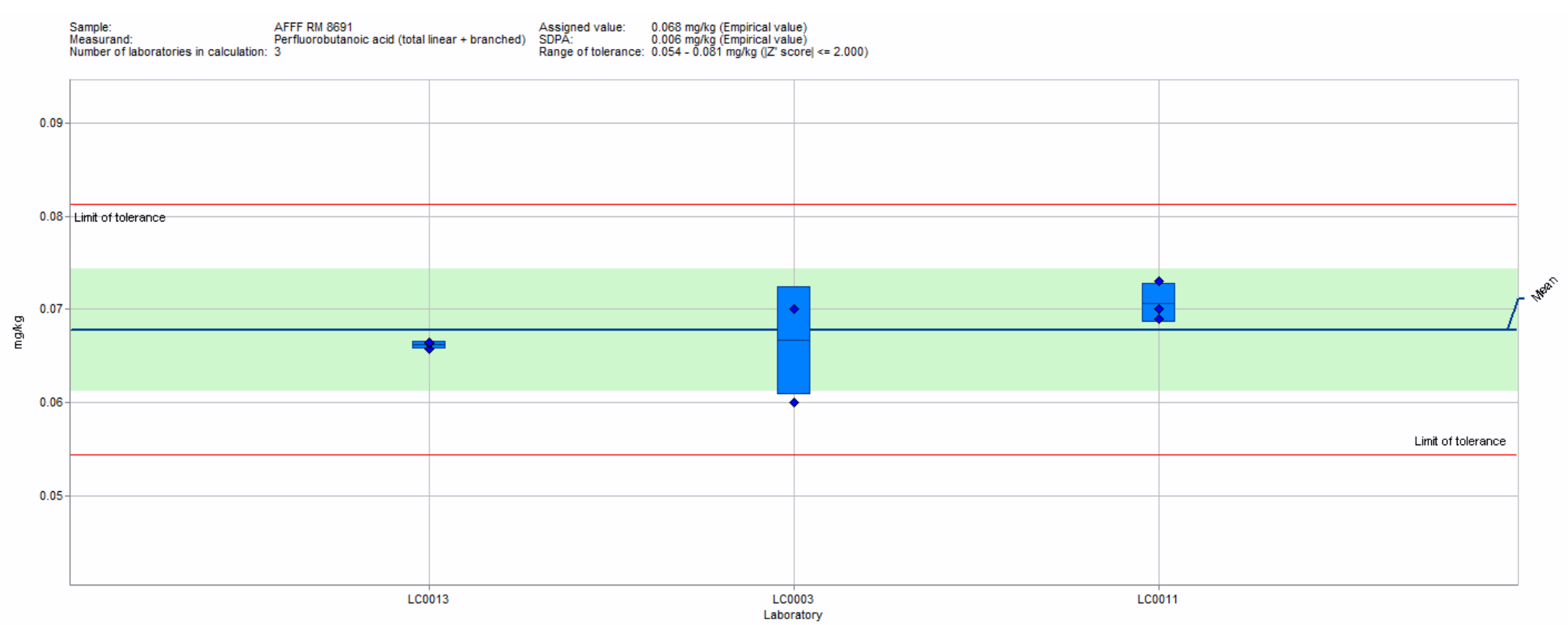

Figure 1-2. PFBA in candidate RM 8691 Per- and Polyfluoroalkyl Substances (PFAS) in Aqueous Film-Forming Foams (AFFF) Formulation II. In this view, individual laboratory data are plotted (diamonds) with the individual laboratory standard deviation (rectangle). The solid blue line represents the consensus mean, and the green shaded region represents the $95 \%$ confidence interval for the consensus mean. The red solid lines represent the consensus range of tolerance, calculated as the values above and below the consensus mean that result in an acceptable $\mathrm{Z}_{\text {comm }}^{\prime}$ score, $\left|\mathrm{Z}_{\text {comm }}^{\prime}\right| \leq 2$. 


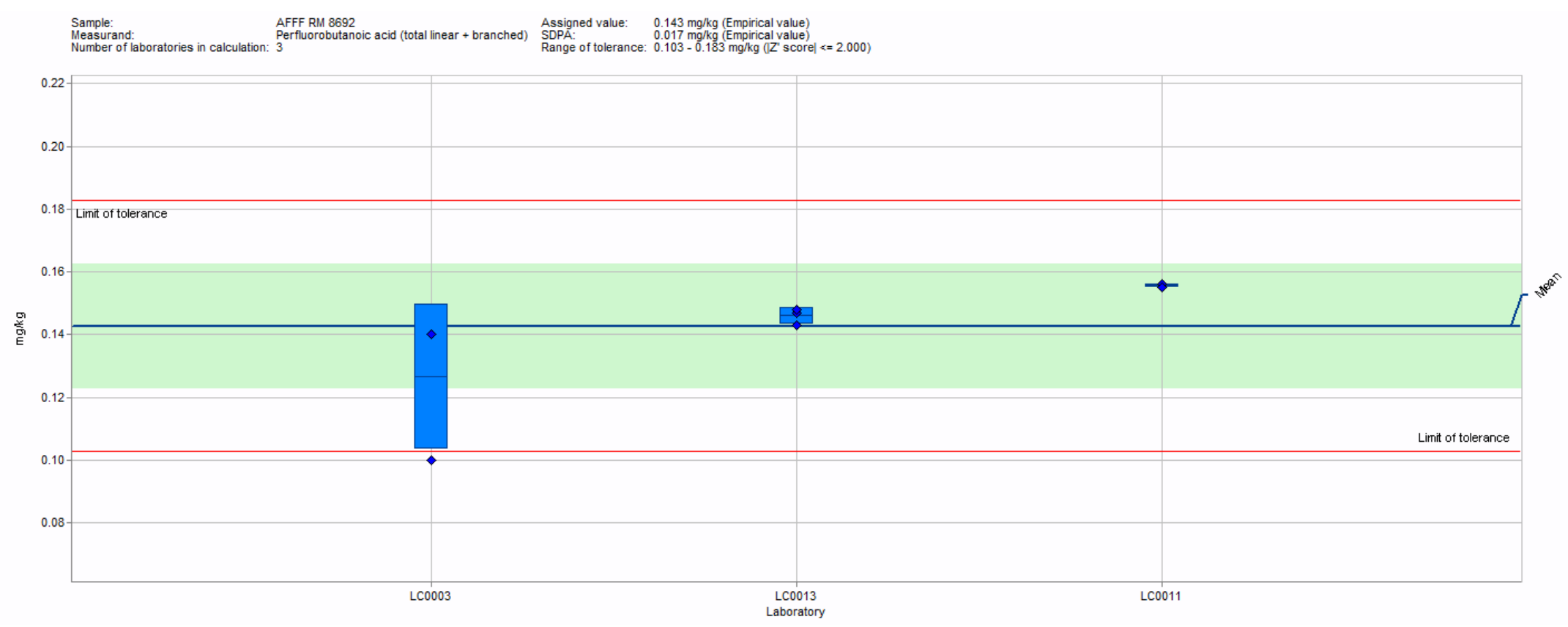

Figure 1-3. PFBA in candidate RM 8692 Per- and Polyfluoroalkyl Substances (PFAS) in Aqueous Film-Forming Foams (AFFF) Formulation III. In this view, individual laboratory data are plotted (diamonds) with the individual laboratory standard deviation (rectangle). The solid blue line represents the consensus mean, and the green shaded region represents the $95 \%$ confidence interval for the consensus mean. The red solid lines represent the consensus range of tolerance, calculated as the values above and below the consensus mean that result in an acceptable $\mathrm{Z}_{\text {comm }}^{\prime}$ score, $\left|\mathrm{Z}_{\text {comm }}^{\prime}\right| \leq 2$. 


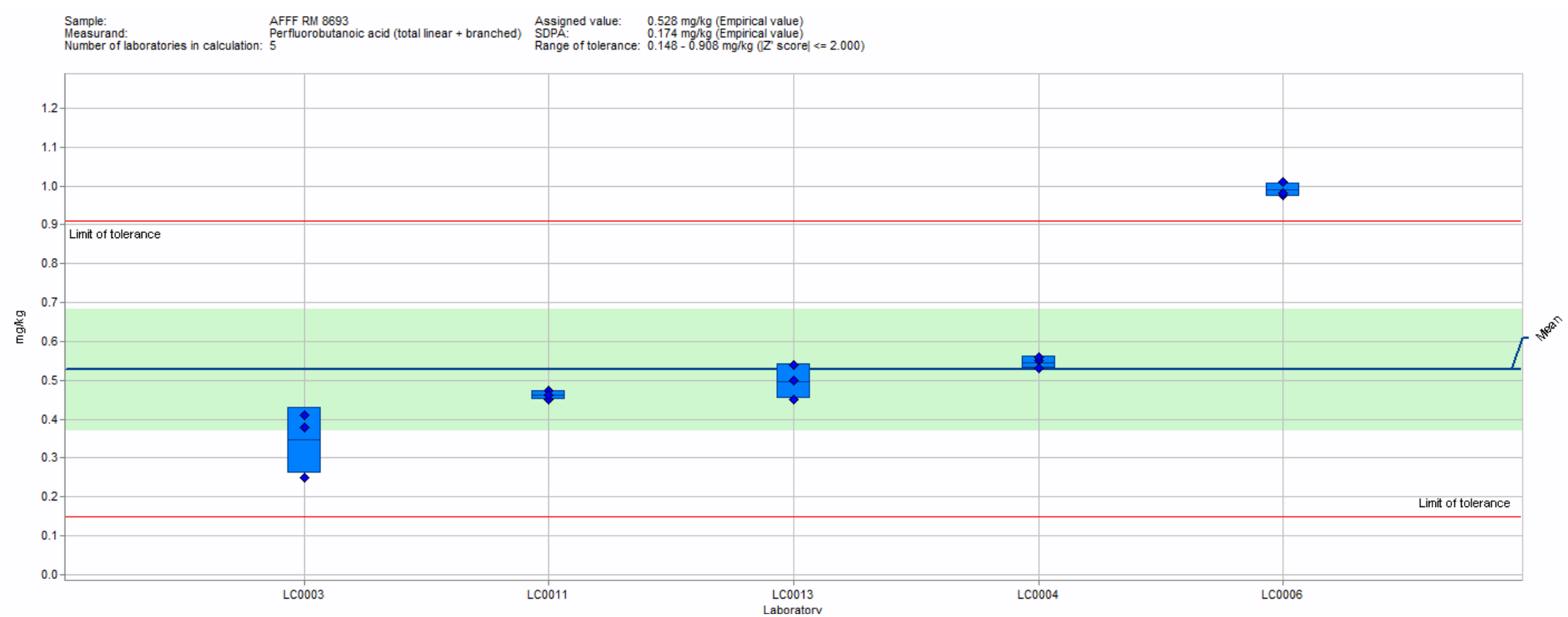

Figure 1-4. PFBA in candidate RM 8693 Per- and Polyfluoroalkyl Substances (PFAS) in Aqueous Film-Forming Foams (AFFF) Formulation IV. In this view, individual laboratory data are plotted (diamonds) with the individual laboratory standard deviation (rectangle). The solid blue line represents the consensus mean, and the green shaded region represents the $95 \%$ confidence interval for the consensus mean. The red solid lines represent the consensus range of tolerance, calculated as the values above and below the consensus mean that result in an acceptable $\mathrm{Z}_{\text {comm }}^{\prime}$ score, $\left|\mathrm{Z}_{\text {comm }}^{\prime}\right| \leq 2$. 


\section{Perfluoropentanoic acid (PFPeA)}

- Six laboratories reported results for PFPeA in candidate RMs 8690, 8691, and 8693. Four laboratories reported results for PFPeA in candidate RM 8692.

- $\quad$ The between-laboratory variability was good for candidate RMs 8690 (12\% RSD)

- The between-laboratory variability for candidate RMs 8691, 8692, and 8693 was poor (53\%, $88 \%$, and $40 \%$ RSD, respectively).

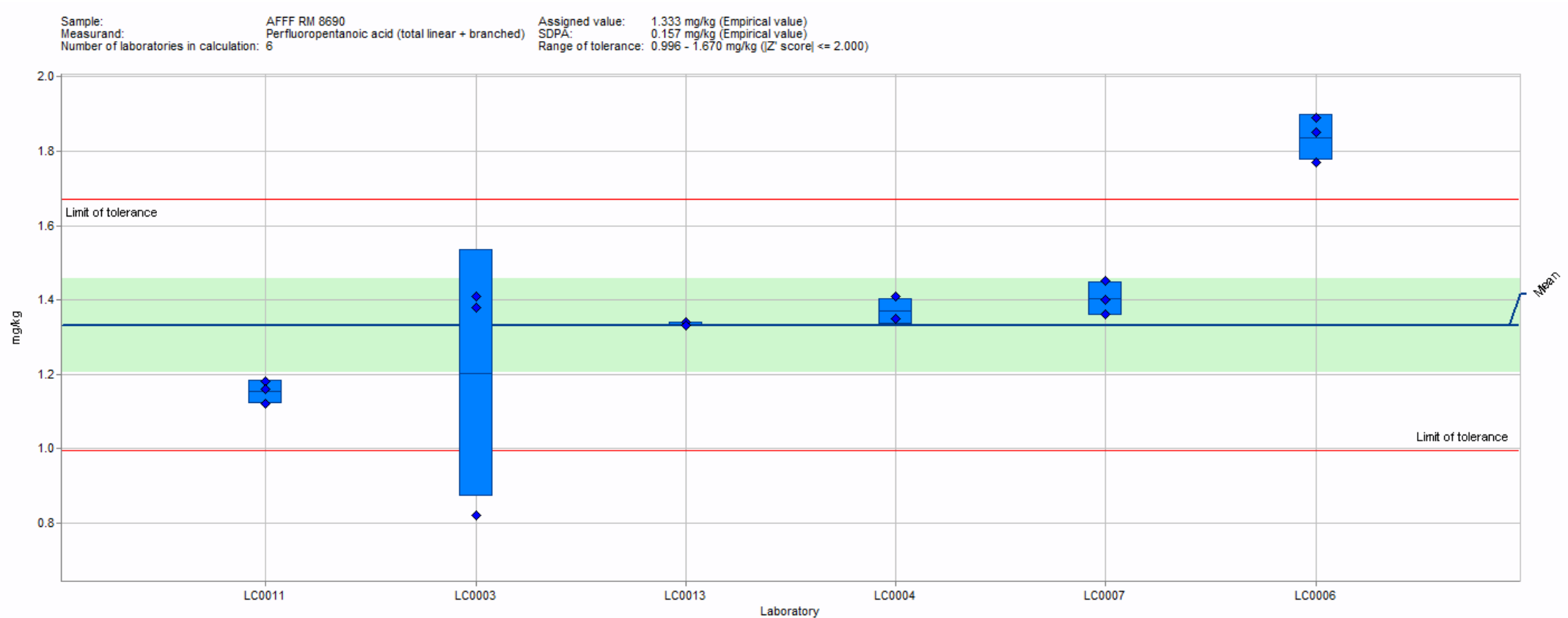

Figure 2-1. PFPeA in candidate RM 8690 Per- and Polyfluoroalkyl Substances (PFAS) in Aqueous Film-Forming Foams (AFFF) Formulation I. In this view, individual laboratory data are plotted (diamonds) with the individual laboratory standard deviation (rectangle). The solid blue line represents the consensus mean, and the green shaded region represents the $95 \%$ confidence interval for the consensus mean. The red solid lines represent the consensus range of tolerance, calculated as the values above and below the consensus mean that result in an acceptable $\mathrm{Z}_{\text {comm }}^{\prime}$ score, $\left|\mathrm{Z}_{\text {comm }}^{\prime}\right| \leq 2$. 


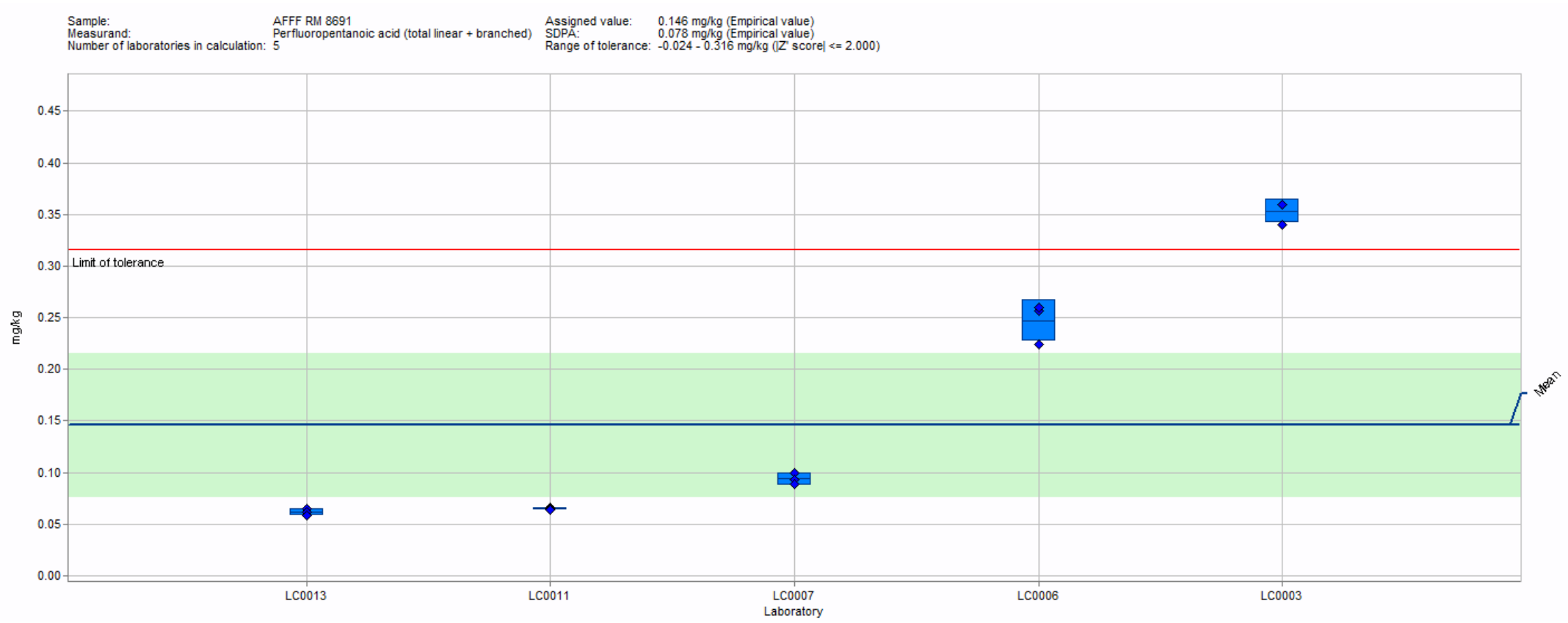

Figure 2-2. PFPeA in candidate RM 8691 Per- and Polyfluoroalkyl Substances (PFAS) in Aqueous Film-Forming Foams (AFFF) Formulation II. In this view, individual laboratory data are plotted (diamonds) with the individual laboratory standard deviation (rectangle). The solid blue line represents the consensus mean, and the green shaded region represents the $95 \%$ confidence interval for the consensus mean. The red solid lines represent the consensus range of tolerance, calculated as the values above and below the consensus mean that result in an acceptable $\mathrm{Z}_{\text {comm }}^{\prime}$ score, $\left|\mathrm{Z}_{\text {comm }}^{\prime}\right| \leq 2$. 


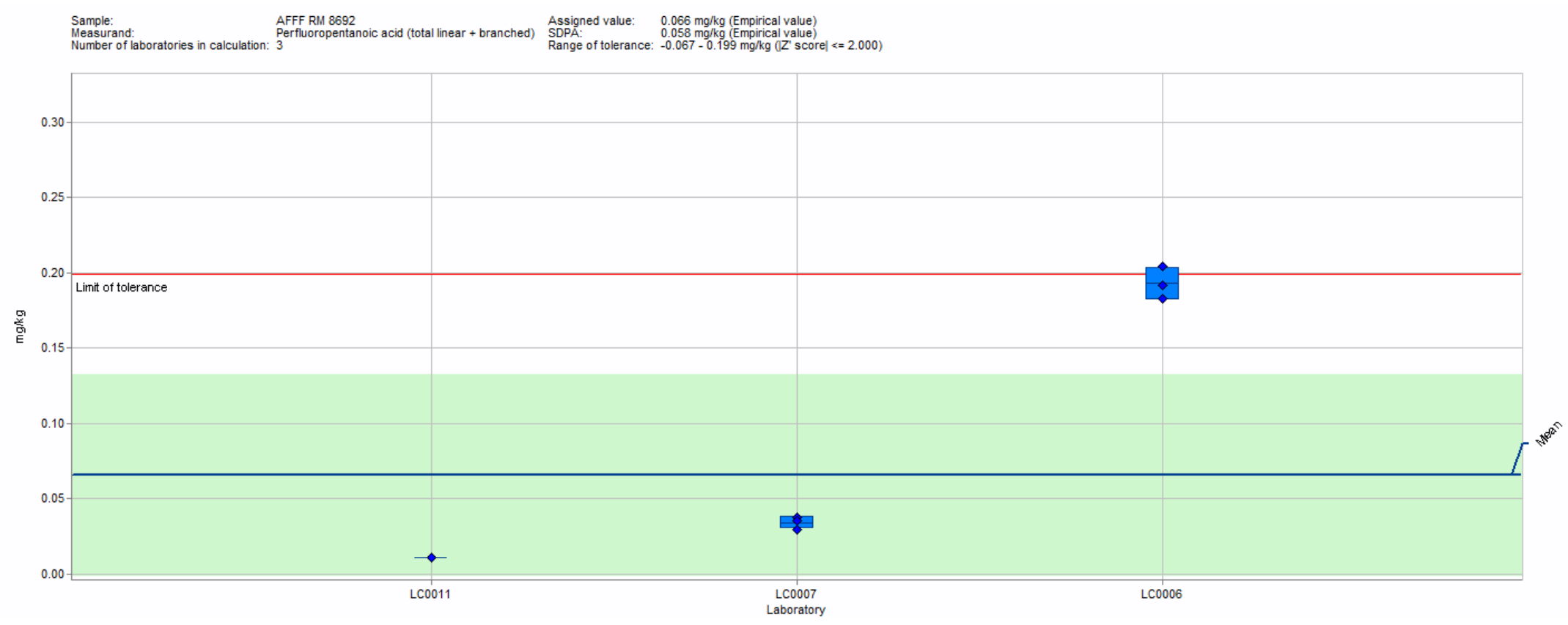

Figure 2-3. PFPeA in candidate RM 8692 Per- and Polyfluoroalkyl Substances (PFAS) in Aqueous Film-Forming Foams (AFFF) Formulation III. In this view, individual laboratory data are plotted (diamonds) with the individual laboratory standard deviation (rectangle). The solid blue line represents the consensus mean, and the green shaded region represents the $95 \%$ confidence interval for the consensus mean. The red solid lines represent the consensus range of tolerance, calculated as the values above and below the consensus mean that result in an acceptable $\mathrm{Z}_{\text {comm }}^{\prime}$ score, $\left|\mathrm{Z}_{\text {comm }}^{\prime}\right| \leq 2$. 


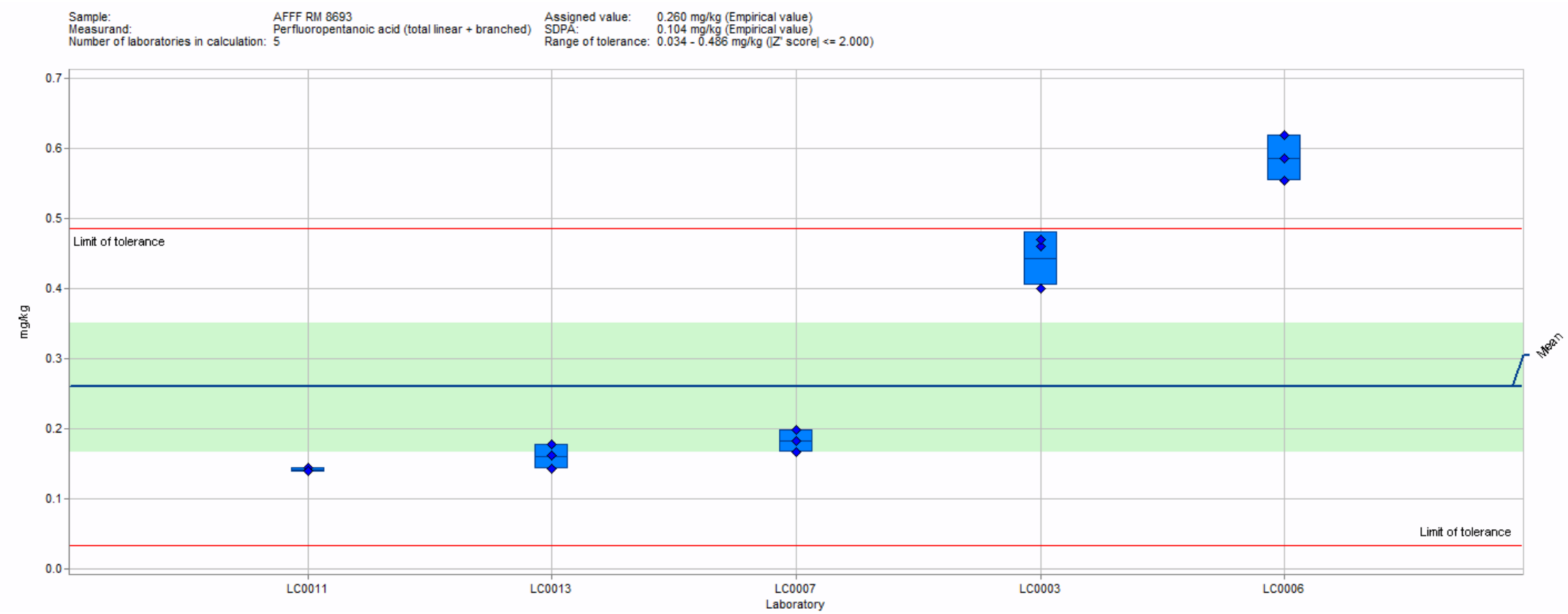

Figure 2-4. PFPeA in candidate RM 8693 Per- and Polyfluoroalkyl Substances (PFAS) in Aqueous Film-Forming Foams (AFFF) Formulation IV. In this view, individual laboratory data are plotted (diamonds) with the individual laboratory standard deviation (rectangle). The solid blue line represents the consensus mean, and the green shaded region represents the $95 \%$ confidence interval for the consensus mean. The red solid lines represent the consensus range of tolerance, calculated as the values above and below the consensus mean that result in an acceptable $\mathrm{Z}_{\text {comm }}^{\prime}$ score, $\left|\mathrm{Z}_{\text {comm }}^{\prime}\right| \leq 2$. 


\section{Perfluorohexanoic acid (PFHxA)}

- Six laboratories reported results for PFHxA in candidate RMs 8690, 8691, and 8693. Five laboratories reported results for PFHxA in candidate RM 8692.

- $\quad$ The between-laboratory variability was good for candidate RM 8690 (13\% RSD).

- The between-laboratory variability was poor for candidate RMs 8691, 8692, and 8693 (24\%, $21 \%$, and $33 \%$ RSD, respectively).

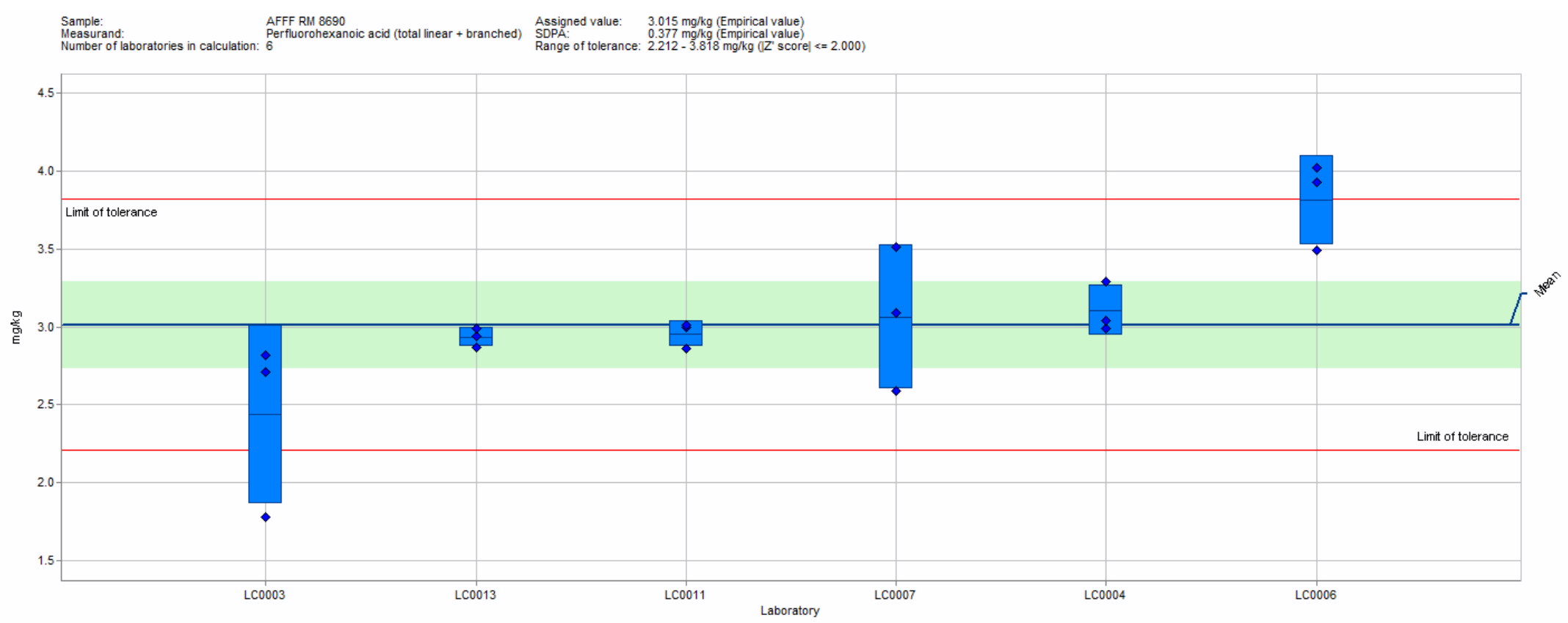

Figure 3-1. PFHxA in candidate RM 8690 Per- and Polyfluoroalkyl Substances (PFAS) in Aqueous Film-Forming Foams (AFFF) Formulation I. In this view, individual laboratory data are plotted (diamonds) with the individual laboratory standard deviation (rectangle). The solid blue line represents the consensus mean, and the green shaded region represents the $95 \%$ confidence interval for the consensus mean. The red solid lines represent the consensus range of tolerance, calculated as the values above and below the consensus mean that result in an acceptable $\mathrm{Z}_{\text {comm }}^{\prime}$ score, $\left|\mathrm{Z}_{\text {comm }}^{\prime}\right| \leq 2$. 


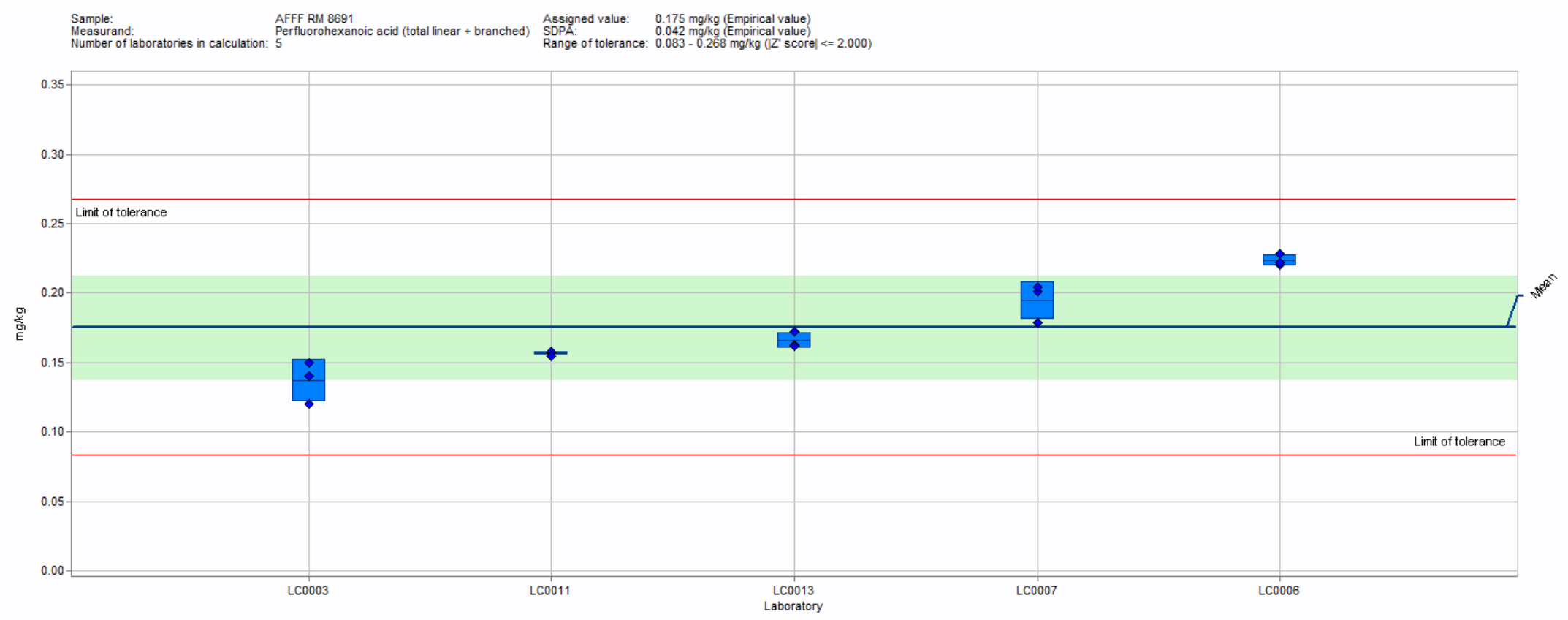

Figure 3-2. PFHxA in candidate RM 8691 Per- and Polyfluoroalkyl Substances (PFAS) in Aqueous Film-Forming Foams (AFFF) Formulation II. In this view, individual laboratory data are plotted (diamonds) with the individual laboratory standard deviation (rectangle). The solid blue line represents the consensus mean, and the green shaded region represents the $95 \%$ confidence interval for the consensus mean. The red solid lines represent the consensus range of tolerance, calculated as the values above and below the consensus mean that result in an acceptable $\mathrm{Z}_{\text {comm }}^{\prime}$ score, $\left|\mathrm{Z}_{\text {comm }}^{\prime}\right| \leq 2$. 


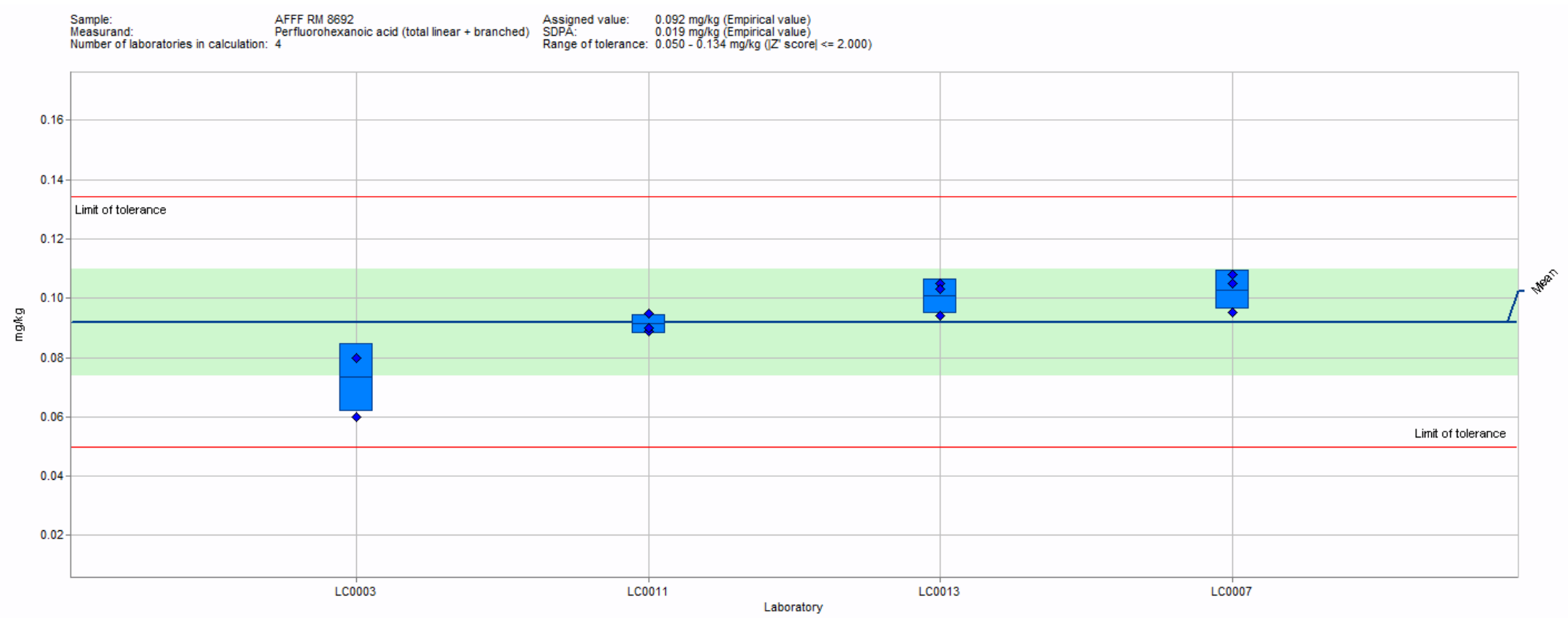

Figure 3-3. PFHxA in candidate RM 8692 Per- and Polyfluoroalkyl Substances (PFAS) in Aqueous Film-Forming Foams (AFFF) Formulation III. In this view, individual laboratory data are plotted (diamonds) with the individual laboratory standard deviation (rectangle). The solid blue line represents the consensus mean, and the green shaded region represents the $95 \%$ confidence interval for the consensus mean. The red solid lines represent the consensus range of tolerance, calculated as the values above and below the consensus mean that result in an acceptable $\mathrm{Z}_{\text {comm }}^{\prime}$ score, $\left|\mathrm{Z}_{\text {comm }}^{\prime}\right| \leq 2$. 


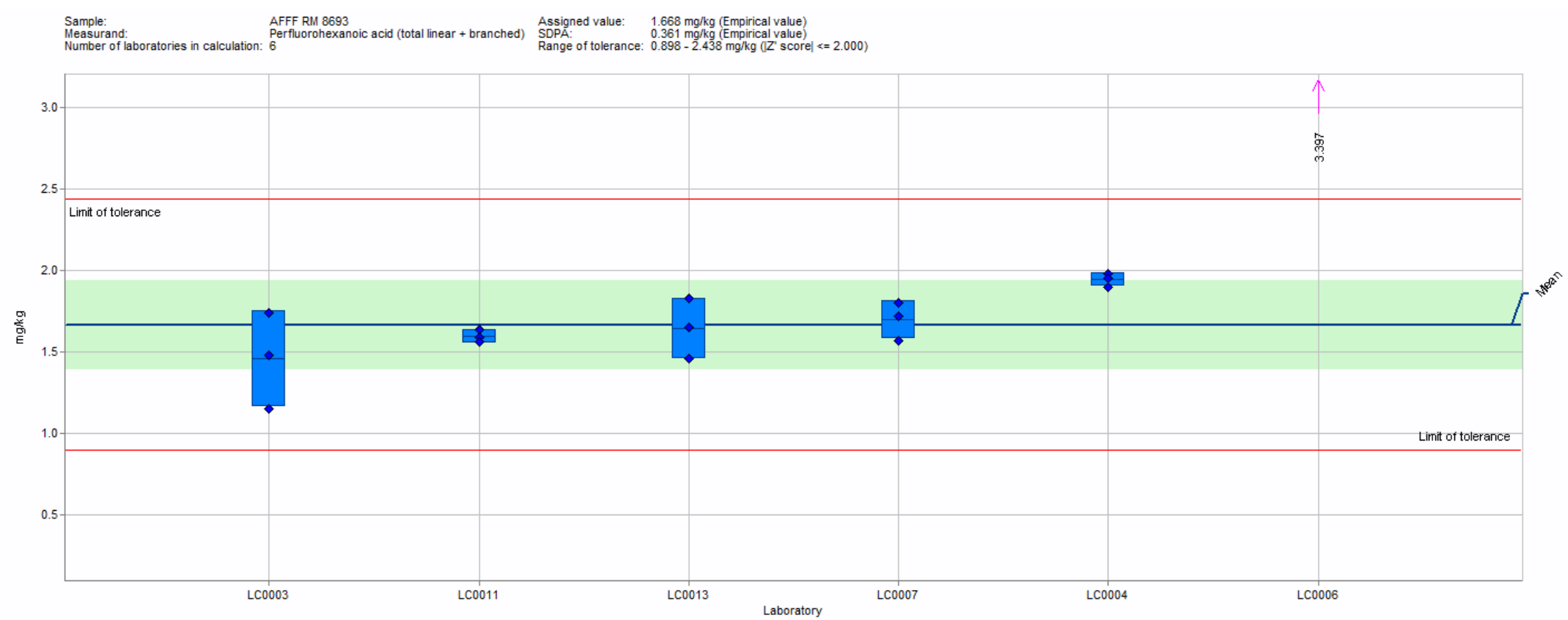

Figure 3-4. PFHxA in candidate RM 8693 Per- and Polyfluoroalkyl Substances (PFAS) in Aqueous Film-Forming Foams (AFFF) Formulation IV. In this view, individual laboratory data are plotted (diamonds) with the individual laboratory standard deviation (rectangle). The solid blue line represents the consensus mean, and the green shaded region represents the $95 \%$ confidence interval for the consensus mean. The red solid lines represent the consensus range of tolerance, calculated as the values above and below the consensus mean that result in an acceptable $Z_{\text {comm }}^{\prime}$ score, $\left|Z_{\text {comm }}^{\prime}\right| \leq 2$. 


\section{Perfluoroheptanoic acid (PFHpA)}

- $\quad$ Six laboratories reported results for PFHpA in candidate RMs 8690 and 8693. Five laboratories reported results for PFHpA in candidate RMs 8691 and 8692.

- $\quad$ The between-laboratory variability was poor for candidate RMs 8690, 8691, 8692, and $8693(39 \%, 83 \%$, $425 \%$, and $100 \%$ RSD, respectively)

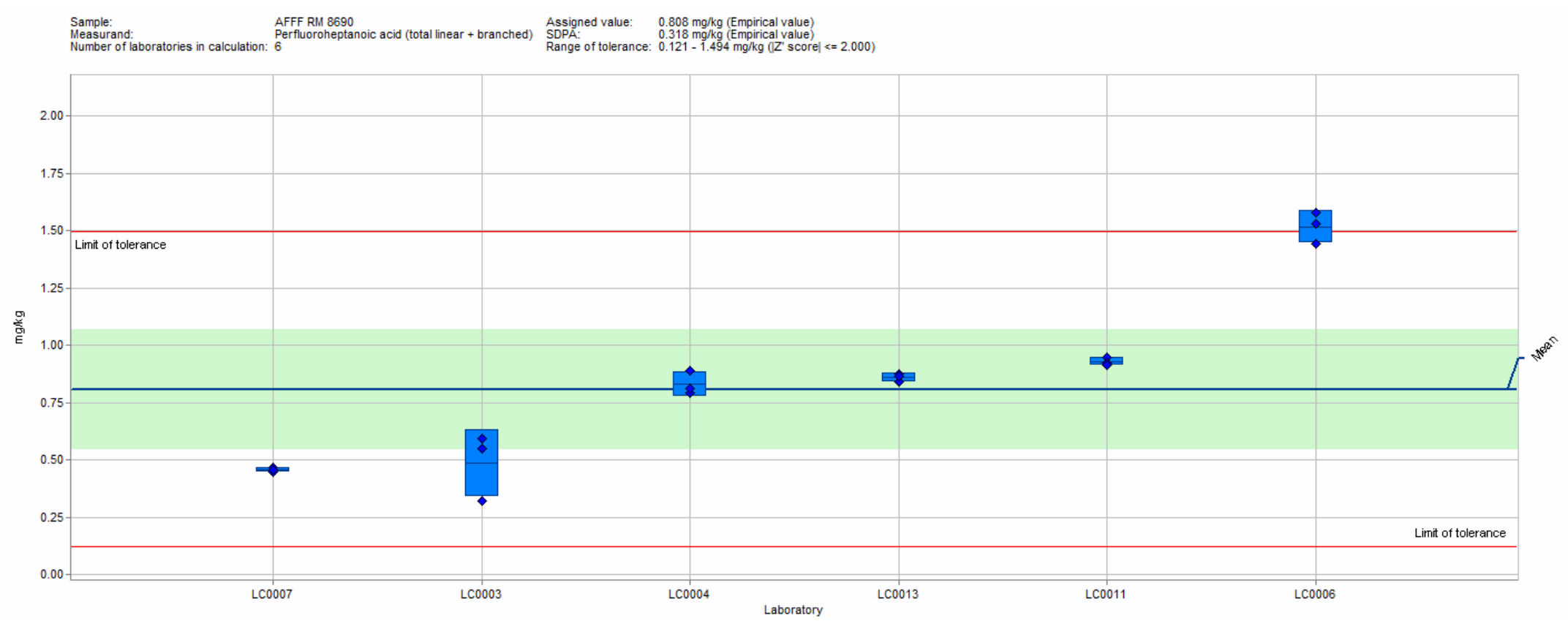

Figure 4-1. PFHpA in candidate RM 8690 Per- and Polyfluoroalkyl Substances (PFAS) in Aqueous Film-Forming Foams (AFFF) Formulation I. In this view, individual laboratory data are plotted (diamonds) with the individual laboratory standard deviation (rectangle). The solid blue line represents the consensus mean, and the green shaded region represents the $95 \%$ confidence interval for the consensus mean. The red solid lines represent the consensus range of tolerance, calculated as the values above and below the consensus mean that result in an acceptable $\mathrm{Z}_{\text {comm }}^{\prime}$ score, $\left|\mathrm{Z}_{\text {comm }}^{\prime}\right| \leq 2$. 


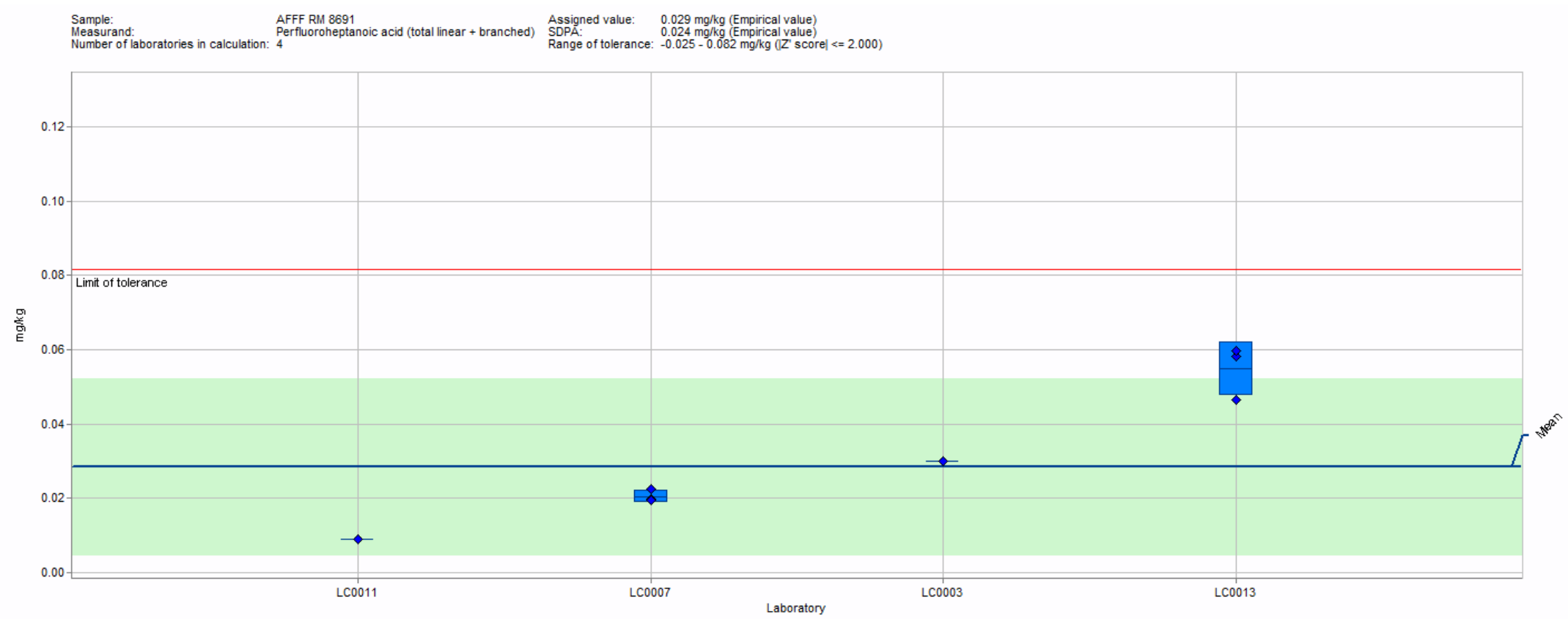

Figure 4-2. PFHpA in candidate RM 8691 Per- and Polyfluoroalkyl Substances (PFAS) in Aqueous Film-Forming Foams (AFFF) Formulation II. In this view, individual laboratory data are plotted (diamonds) with the individual laboratory standard deviation (rectangle). The solid blue line represents the consensus mean, and the green shaded region represents the $95 \%$ confidence interval for the consensus mean. The red solid lines represent the consensus range of tolerance, calculated as the values above and below the consensus mean that result in an acceptable $\mathrm{Z}_{\text {comm }}^{\prime}$ score, $\left|\mathrm{Z}_{\text {comm }}^{\prime}\right| \leq 2$. 


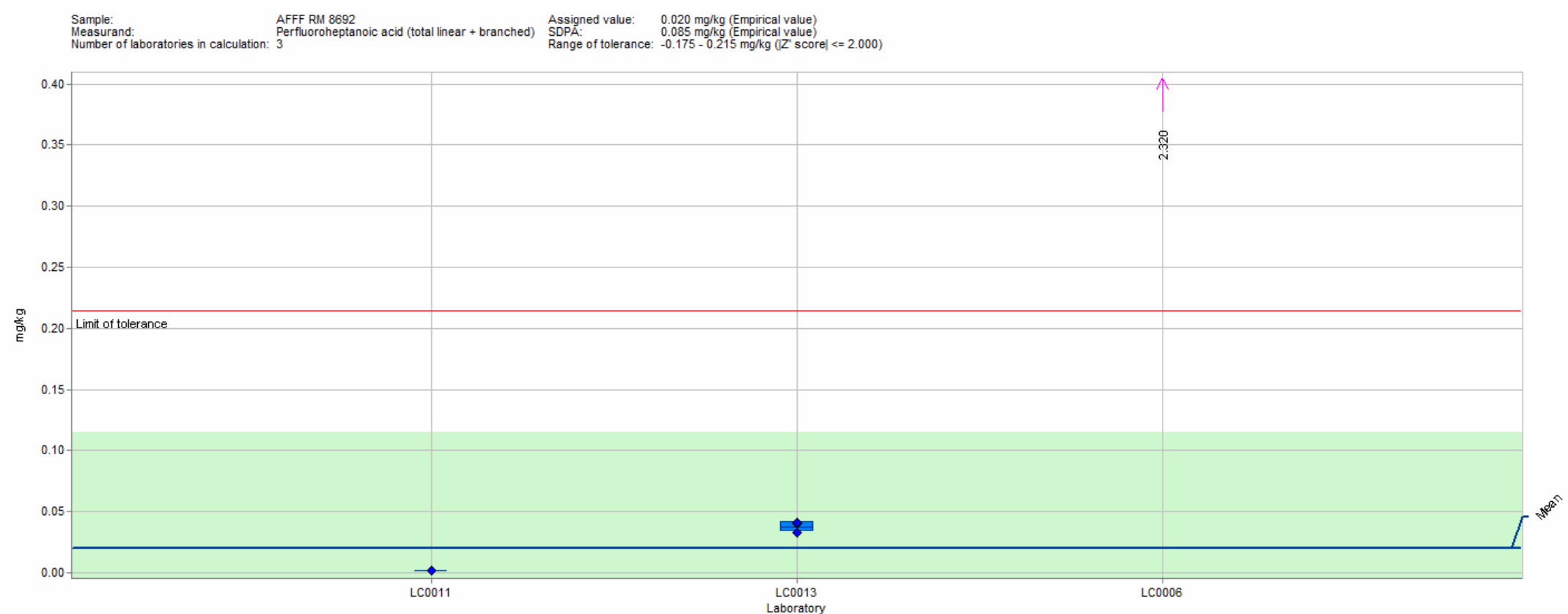

Figure 4-3. PFHpA in candidate RM 8692 Per- and Polyfluoroalkyl Substances (PFAS) in Aqueous Film-Forming Foams (AFFF) Formulation III. In this view, individual laboratory data are plotted (diamonds) with the individual laboratory standard deviation (rectangle). The solid blue line represents the consensus mean, and the green shaded region represents the $95 \%$ confidence interval for the consensus mean. The red solid lines represent the consensus range of tolerance, calculated as the values above and below the consensus mean that result in an acceptable $\mathrm{Z}_{\text {comm }}^{\prime}$ score, $\left|\mathrm{Z}_{\text {comm }}^{\prime}\right| \leq 2$. 


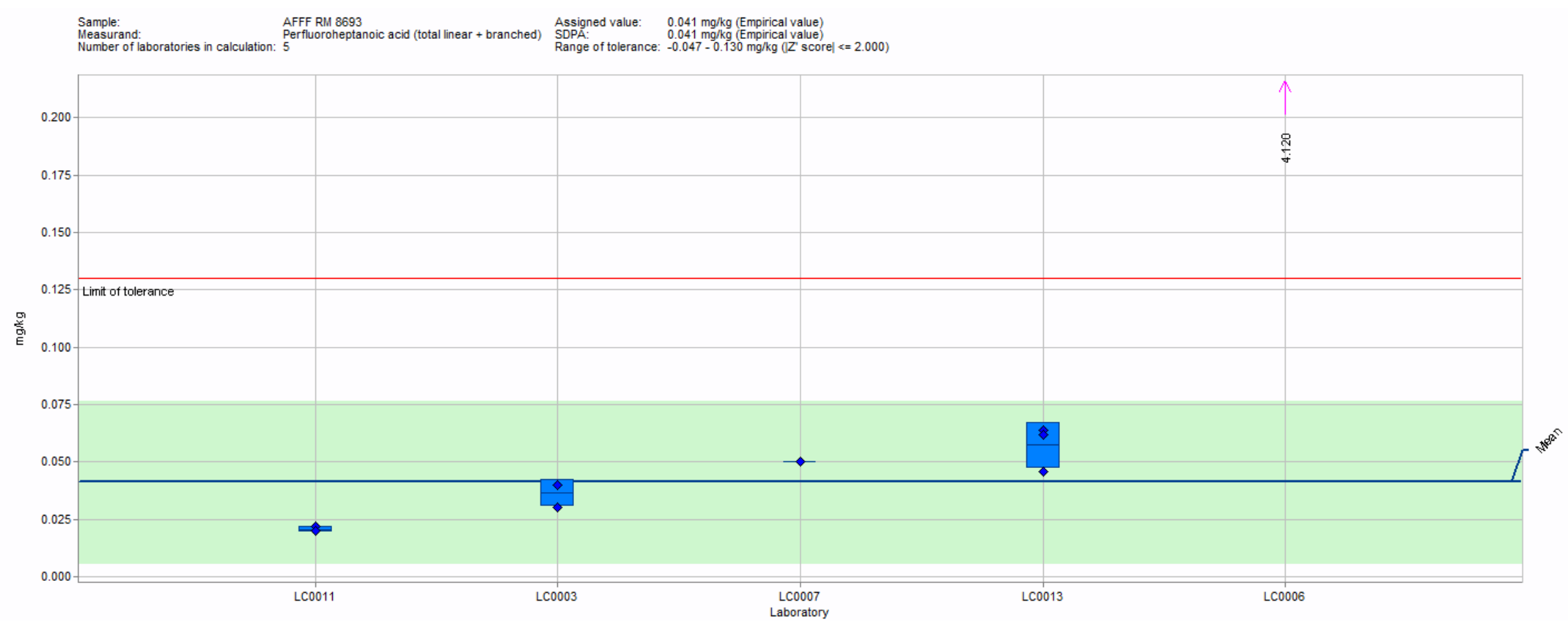

Figure 4-4. PFHpA in candidate RM 8693 Per- and Polyfluoroalkyl Substances (PFAS) in Aqueous Film-Forming Foams (AFFF) Formulation IV. In this view, individual laboratory data are plotted (diamonds) with the individual laboratory standard deviation (rectangle). The solid blue line represents the consensus mean, and the green shaded region represents the $95 \%$ confidence interval for the consensus mean. The red solid lines represent the consensus range of tolerance, calculated as the values above and below the consensus mean that result in an acceptable $\mathrm{Z}_{\text {comm }}^{\prime}$ score, $\left|\mathrm{Z}_{\text {comm }}^{\prime}\right| \leq 2$. 
- Six laboratories reported results for PFOA in candidate RM 8690. Five laboratories reported results for PFOA in candidate RM 8691

- The between-laboratory variability was poor for candidate RMs 8690 and 8691 (29\% and $42 \%$ RSD, respectively)

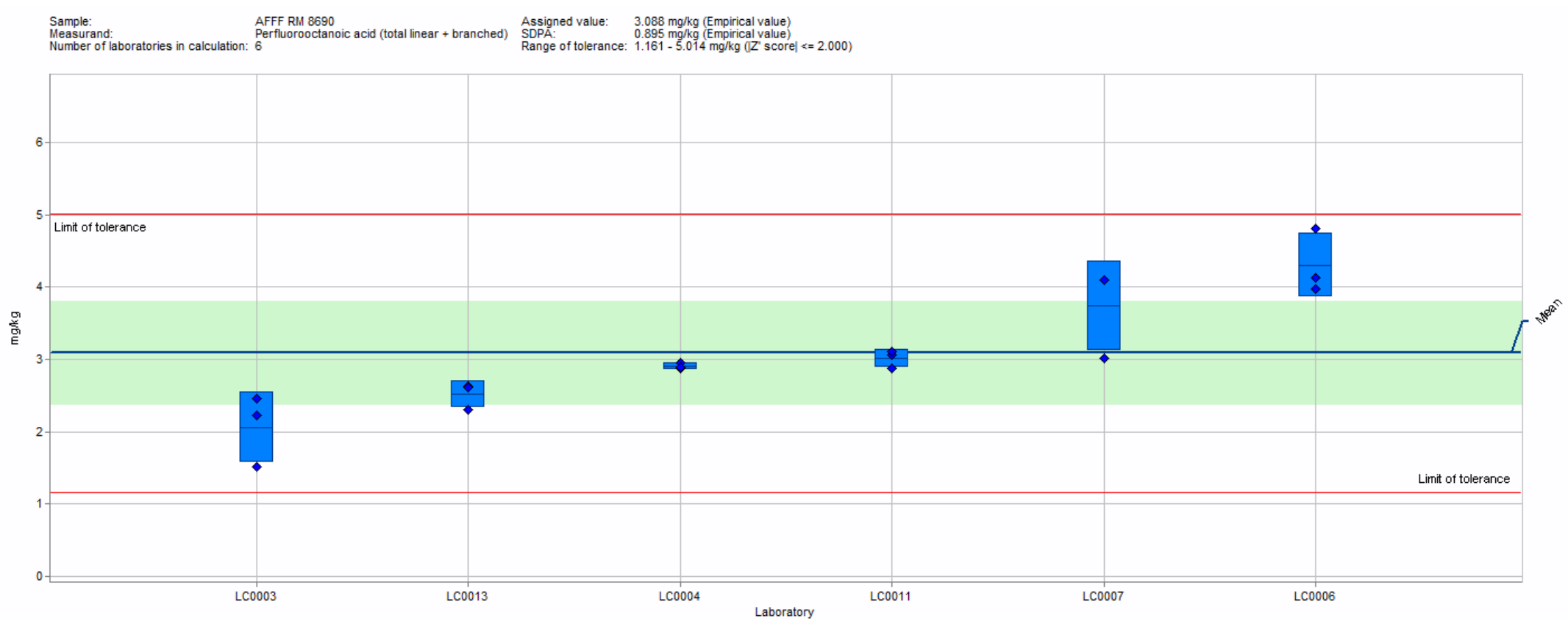

Figure 5-1. PFOA in candidate RM 8690 Per- and Polyfluoroalkyl Substances (PFAS) in Aqueous Film-Forming Foams (AFFF) Formulation I. In this view, individual laboratory data are plotted (diamonds) with the individual laboratory standard deviation (rectangle). The solid blue line represents the consensus mean, and the green shaded region represents the $95 \%$ confidence interval for the consensus mean. The red solid lines represent the consensus range of tolerance, calculated as the values above and below the consensus mean that result in an acceptable $\mathrm{Z}_{\text {comm }}^{\prime}$ score, $\left|\mathrm{Z}_{\text {comm }}^{\prime}\right| \leq 2$. 


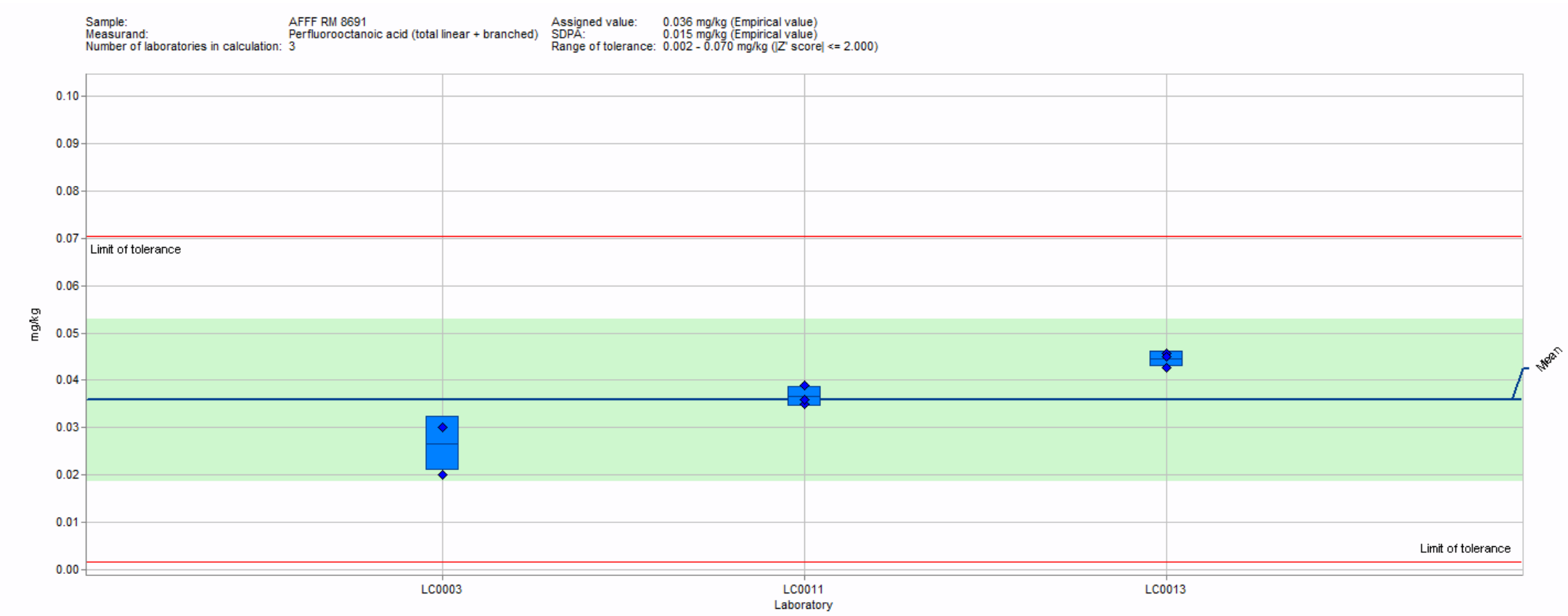

Figure 5-2. PFOA in candidate RM 8691 Per- and Polyfluoroalkyl Substances (PFAS) in Aqueous Film-Forming Foams (AFFF) Formulation II. In this view, individual laboratory data are plotted (diamonds) with the individual laboratory standard deviation (rectangle). The solid blue line represents the consensus mean, and the green shaded region represents the $95 \%$ confidence interval for the consensus mean. The red solid lines represent the consensus range of tolerance, calculated as the values above and below the consensus mean that result in an acceptable $Z_{\text {comm }}^{\prime}$ score, $\left|Z_{\text {comm }}^{\prime}\right| \leq 2$. 


\section{Perfluorononanoic acid (PFNA)}

- $\quad$ Four laboratories reported results for PFNA in candidate RM 8691. Three laboratories reported results for PFNA in candidate RM 8690

- $\quad$ Although laboratories reported results for PFNA, only two laboratories reported results for PFNA above their detection limits

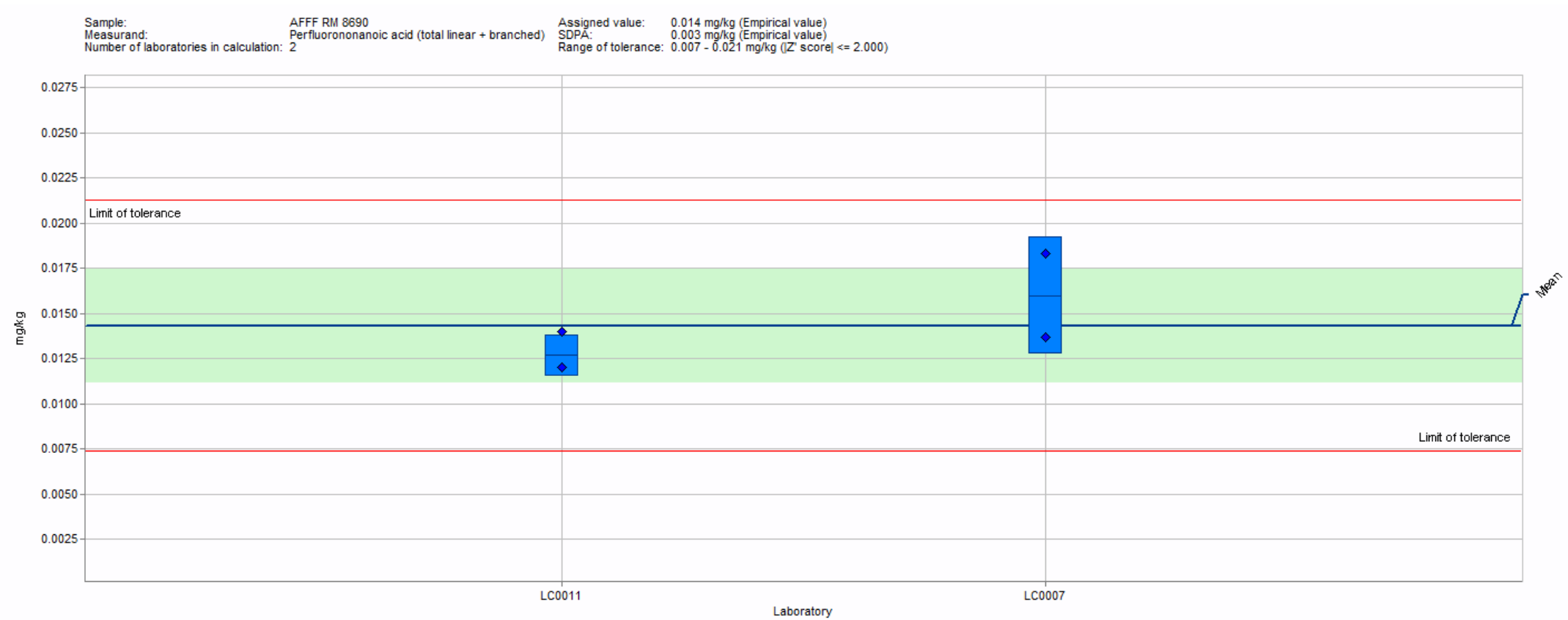

Figure 6-1. PFNA in candidate RM 8690 Per- and Polyfluoroalkyl Substances (PFAS) in Aqueous Film-Forming Foams (AFFF) Formulation I. In this view, individual laboratory data are plotted (diamonds) with the individual laboratory standard deviation (rectangle). The solid blue line represents the consensus mean, and the green shaded region represents the $95 \%$ confidence interval for the consensus mean. The red solid lines represent the consensus range of tolerance, calculated as the values above and below the consensus mean that result in an acceptable $\mathrm{Z}_{\text {comm }}^{\prime}$ score, $\left|\mathrm{Z}_{\text {comm }}^{\prime}\right| \leq 2$. 


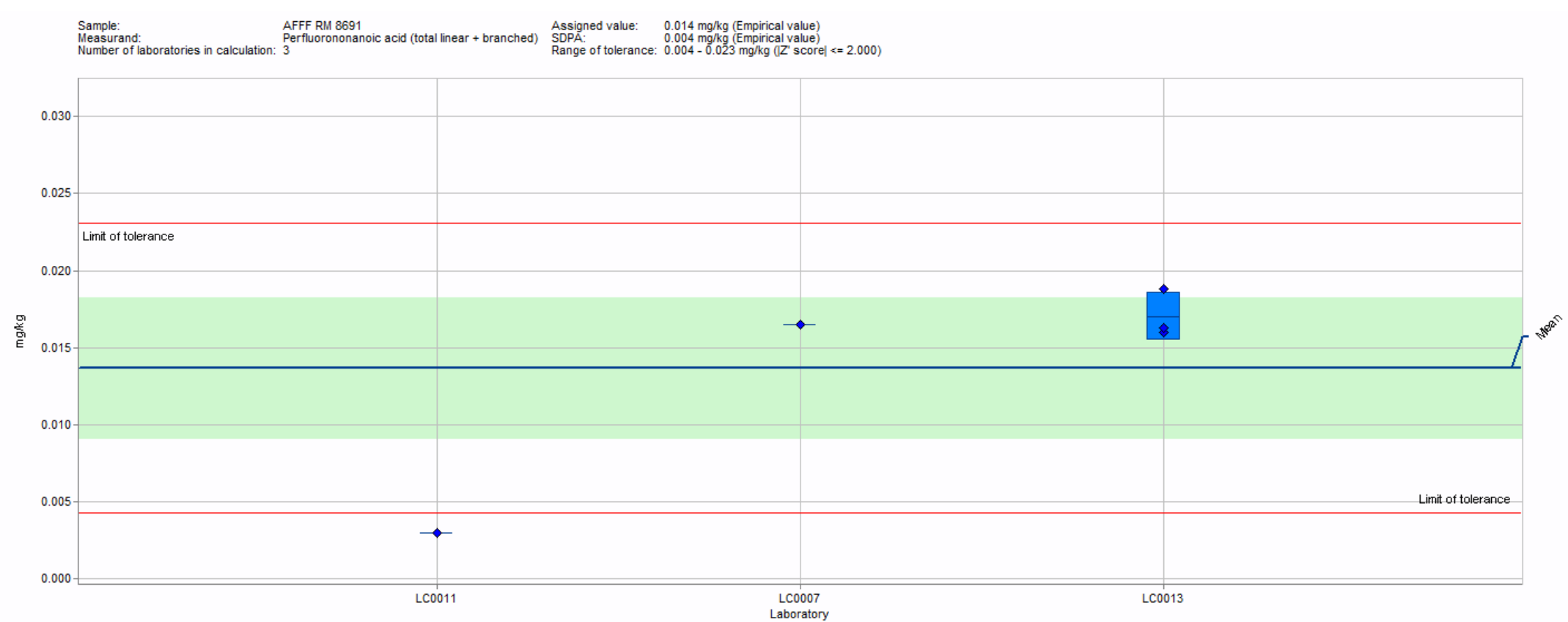

Figure 6-2. PFNA in candidate RM 8691 Per- and Polyfluoroalkyl Substances (PFAS) in Aqueous Film-Forming Foams (AFFF) Formulation II. In this view, individual laboratory data are plotted (diamonds) with the individual laboratory standard deviation (rectangle). The solid blue line represents the consensus mean, and the green shaded region represents the $95 \%$ confidence interval for the consensus mean. The red solid lines represent the consensus range of tolerance, calculated as the values above and below the consensus mean that result in an acceptable $\mathrm{Z}_{\text {comm }}^{\prime}$ score, $\left|\mathrm{Z}_{\text {comm }}^{\prime}\right| \leq 2$. 
Perfluorodecanoic acid (PFDA)

- $\quad$ Five laboratories reported results for PFDA in candidate RM 8691. Four laboratories reported results for PFDA in candidate RM 8690

- $\quad$ The between-laboratory variability was poor for candidate RMs 8690 and 8691 (312\% and $32 \%$ RSD, respectively)

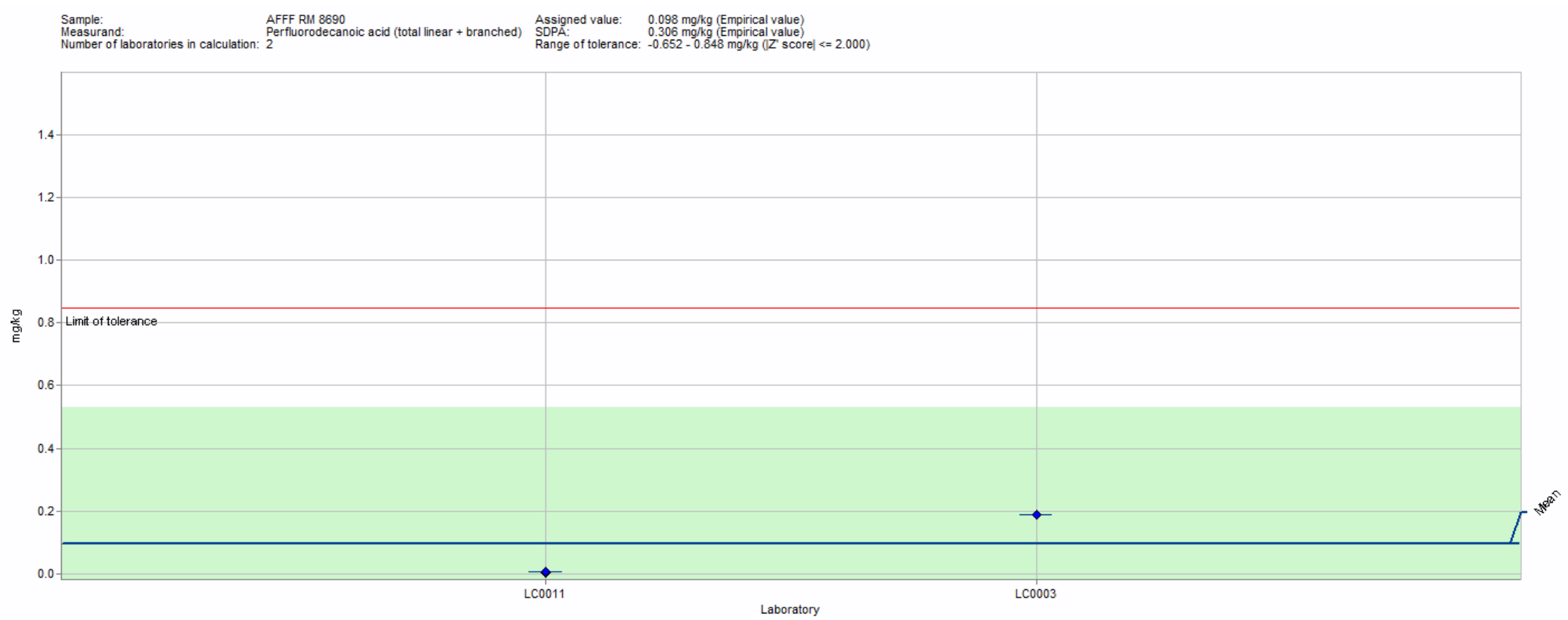

Figure 7-1. PFDA in candidate RM 8690 Per- and Polyfluoroalkyl Substances (PFAS) in Aqueous Film-Forming Foams (AFFF) Formulation I. In this view, individual laboratory data are plotted (diamonds) with the individual laboratory standard deviation (rectangle). The solid blue line represents the consensus mean, and the green shaded region represents the $95 \%$ confidence interval for the consensus mean. The red solid lines represent the consensus range of tolerance, calculated as the values above and below the consensus mean that result in an acceptable $\mathrm{Z}_{\text {comm }}^{\prime}$ score, $\left|\mathrm{Z}_{\text {comm }}^{\prime}\right| \leq 2$. 


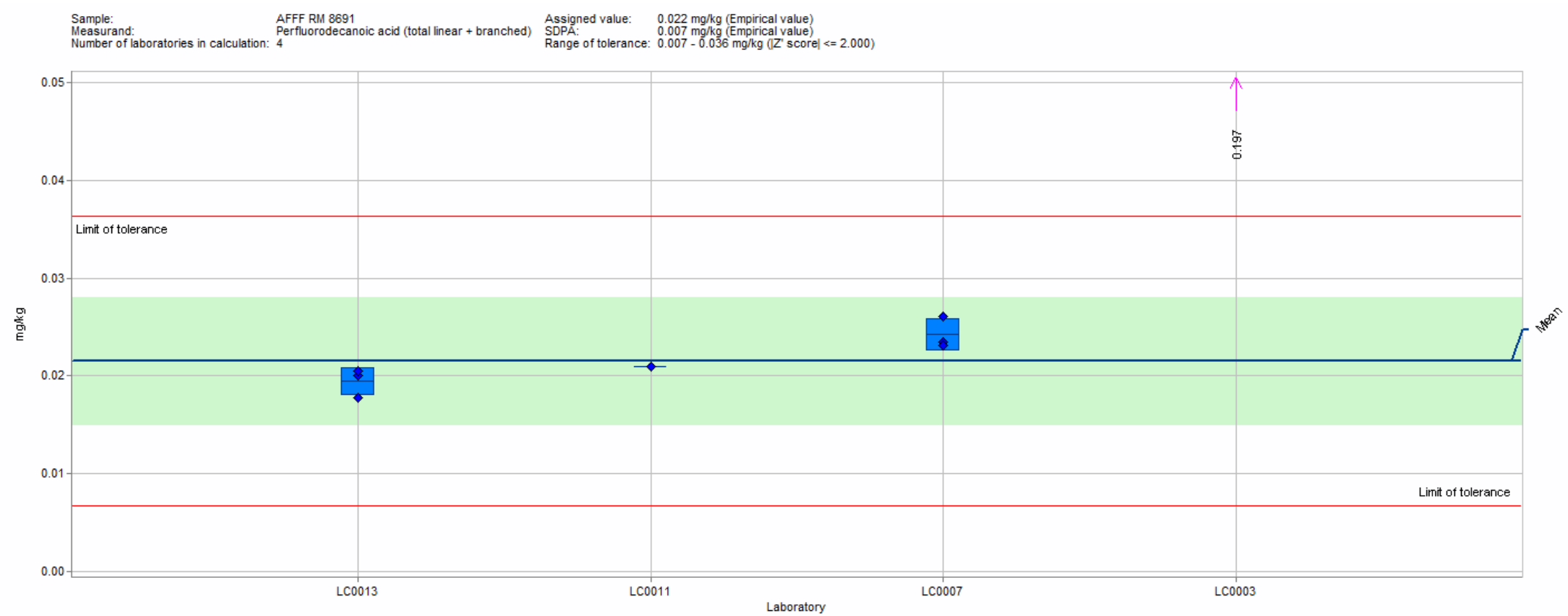

Figure 7-2. PFDA in candidate RM 8691 Per- and Polyfluoroalkyl Substances (PFAS) in Aqueous Film-Forming Foams (AFFF) Formulation II. In this view, individual laboratory data are plotted (diamonds) with the individual laboratory standard deviation (rectangle). The solid blue line represents the consensus mean, and the green shaded region represents the $95 \%$ confidence interval for the consensus mean. The red solid lines represent the consensus range of tolerance, calculated as the values above and below the consensus mean that result in an acceptable $\mathrm{Z}_{\text {comm }}^{\prime}$ score, $\left|\mathrm{Z}_{\text {comm }}^{\prime}\right| \leq 2$. 


\section{Perfluoroundecanoic acid (PFUnA)}

- $\quad$ Three laboratories reported results for PFUnA in candidate RM 8691.

- The between-laboratory variability was poor for candidate RM 8691 (255\% RSD)

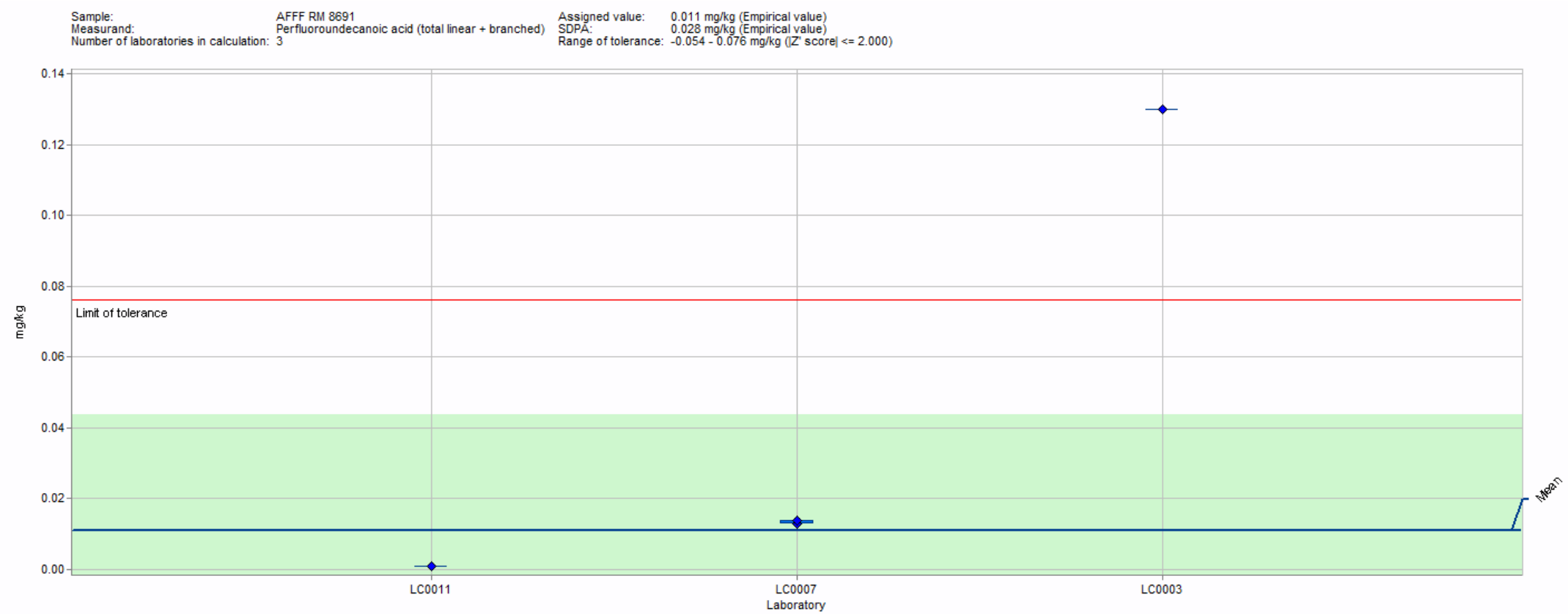

Figure 8. PFUnA in candidate RM 8691 Per- and Polyfluoroalkyl Substances (PFAS) in Aqueous Film-Forming Foams (AFFF) Formulation II. In this view, individual laboratory data are plotted (diamonds) with the individual laboratory standard deviation (rectangle). The solid blue line represents the consensus mean, and the green shaded region represents the $95 \%$ confidence interval for the consensus mean. The red solid lines represent the consensus range of tolerance, calculated as the values above and below the consensus mean that result in an acceptable $\mathrm{Z}_{\text {comm }}^{\prime}$ score, $\left|\mathrm{Z}_{\text {comm }}^{\prime}\right| \leq 2$. 
- $\quad$ Four laboratories reported results for PFTA in candidate RM 8691.

- The between-laboratory variability was poor for candidate RM 8691 (235\% RSD)

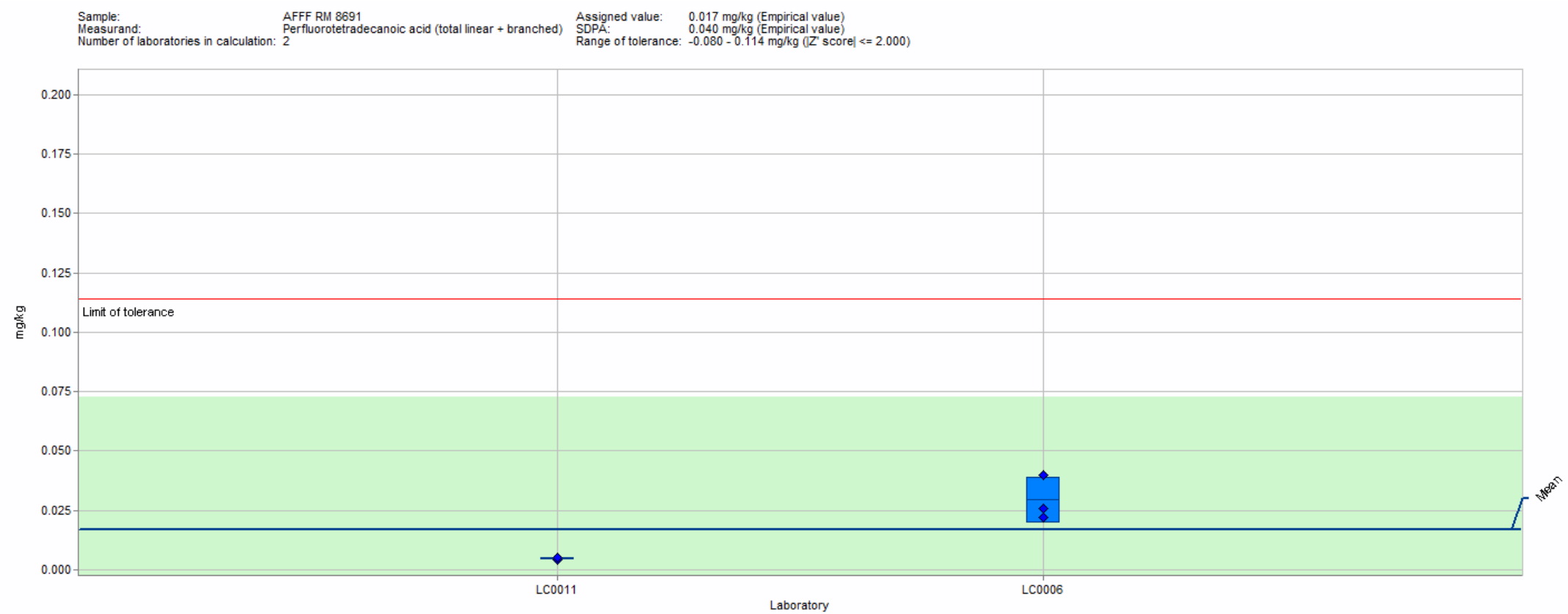

Figure 9. PFTA in candidate RM 8691 Per- and Polyfluoroalkyl Substances (PFAS) in Aqueous Film-Forming Foams (AFFF) Formulation II. In this view, individual laboratory data are plotted (diamonds) with the individual laboratory standard deviation (rectangle). The solid blue line represents the consensus mean, and the green shaded region represents the $95 \%$ confidence interval for the consensus mean. The red solid lines represent the consensus range of tolerance, calculated as the values above and below the consensus mean that result in an acceptable $\mathrm{Z}_{\text {comm }}^{\prime}$ score, $\left|\mathrm{Z}_{\text {comm }}^{\prime}\right| \leq 2$. 


\section{Perfluorobutanesulfonic acid (PFBS)}

- $\quad$ Six laboratories reported results for PFBS in candidate RM 8690. Three laboratories reported results for PFBS in candidate RMs 8691, 8692, and 8693.

- $\quad$ The between-laboratory variability was good for candidate RM 8690 (12\% RSD)

- The between-laboratory variability was poor for candidate RMs 8691, 8692, and 8693 (124\%, $316 \%$, and 410\% RSD, respectively)

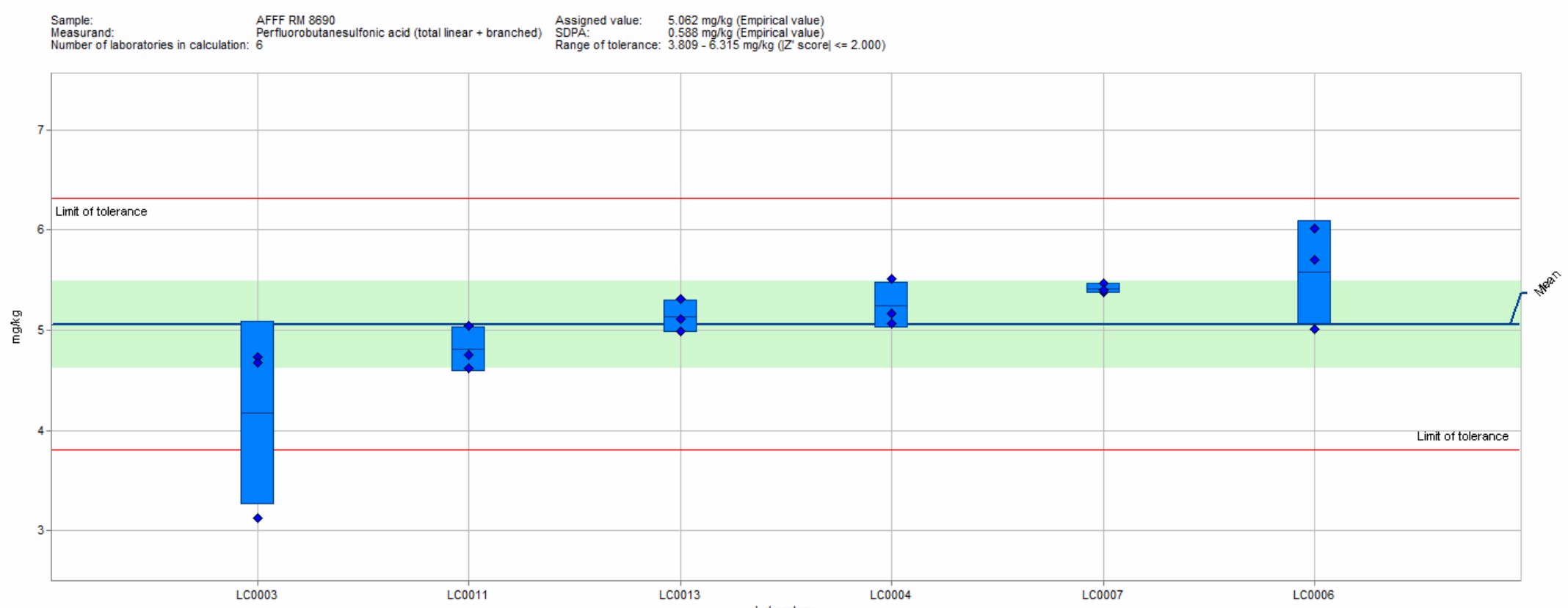

Figure 10-1. PFBS in candidate RM 8690 Per- and Polyfluoroalkyl Substances (PFAS) in Aqueous Film-Forming Foams (AFFF) Formulation I. In this view, individual laboratory data are plotted (diamonds) with the individual laboratory standard deviation (rectangle). The solid blue line represents the consensus mean, and the green shaded region represents the $95 \%$ confidence interval for the consensus mean. The red solid lines represent the consensus range of tolerance, calculated as the values above and below the consensus mean that result in an acceptable $\mathrm{Z}_{\text {comm }}^{\prime}$ score, $\left|\mathrm{Z}_{\text {comm }}^{\prime}\right| \leq 2$. 


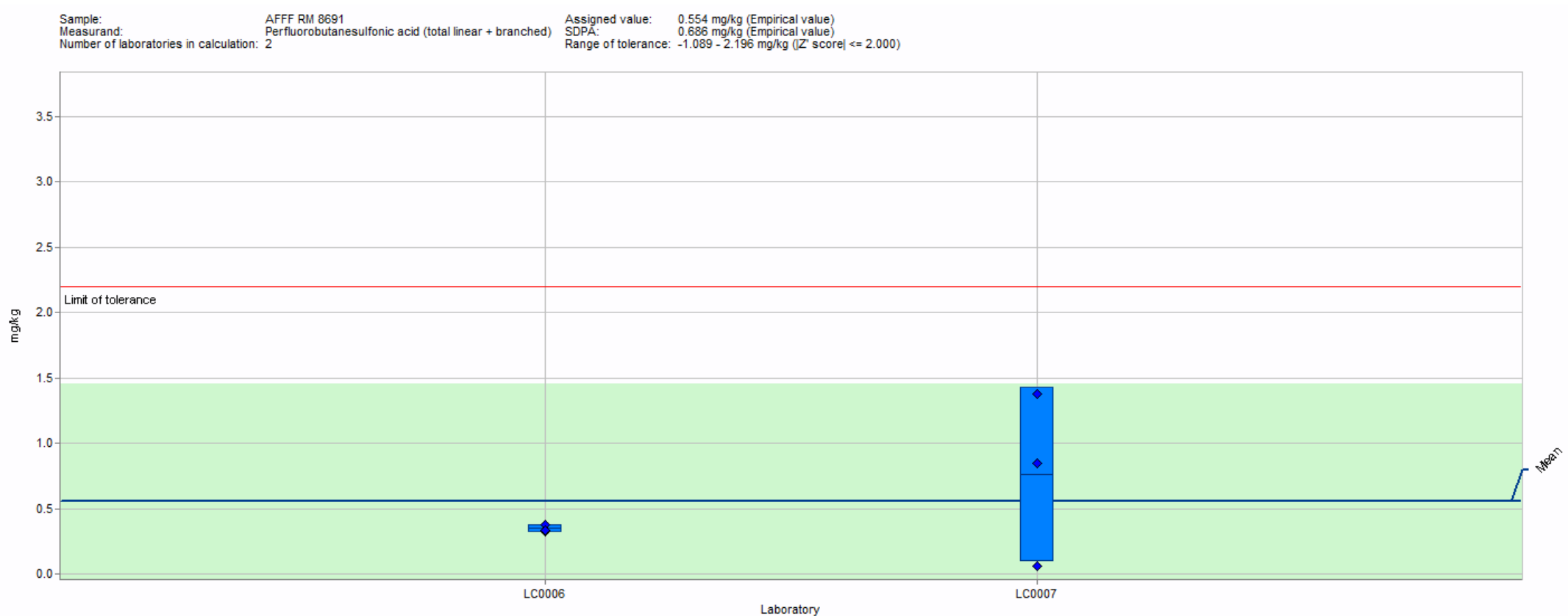

Figure 10-2. PFBS in candidate RM 8691 Per- and Polyfluoroalkyl Substances (PFAS) in Aqueous Film-Forming Foams (AFFF) Formulation II. In this view, individual laboratory data are plotted (diamonds) with the individual laboratory standard deviation (rectangle). The solid blue line represents the consensus mean, and the green shaded region represents the $95 \%$ confidence interval for the consensus mean. The red solid lines represent the consensus range of tolerance, calculated as the values above and below the consensus mean that result in an acceptable $\mathrm{Z}_{\text {comm }}^{\prime}$ score, $\left|\mathrm{Z}_{\text {comm }}^{\prime}\right| \leq 2$. 


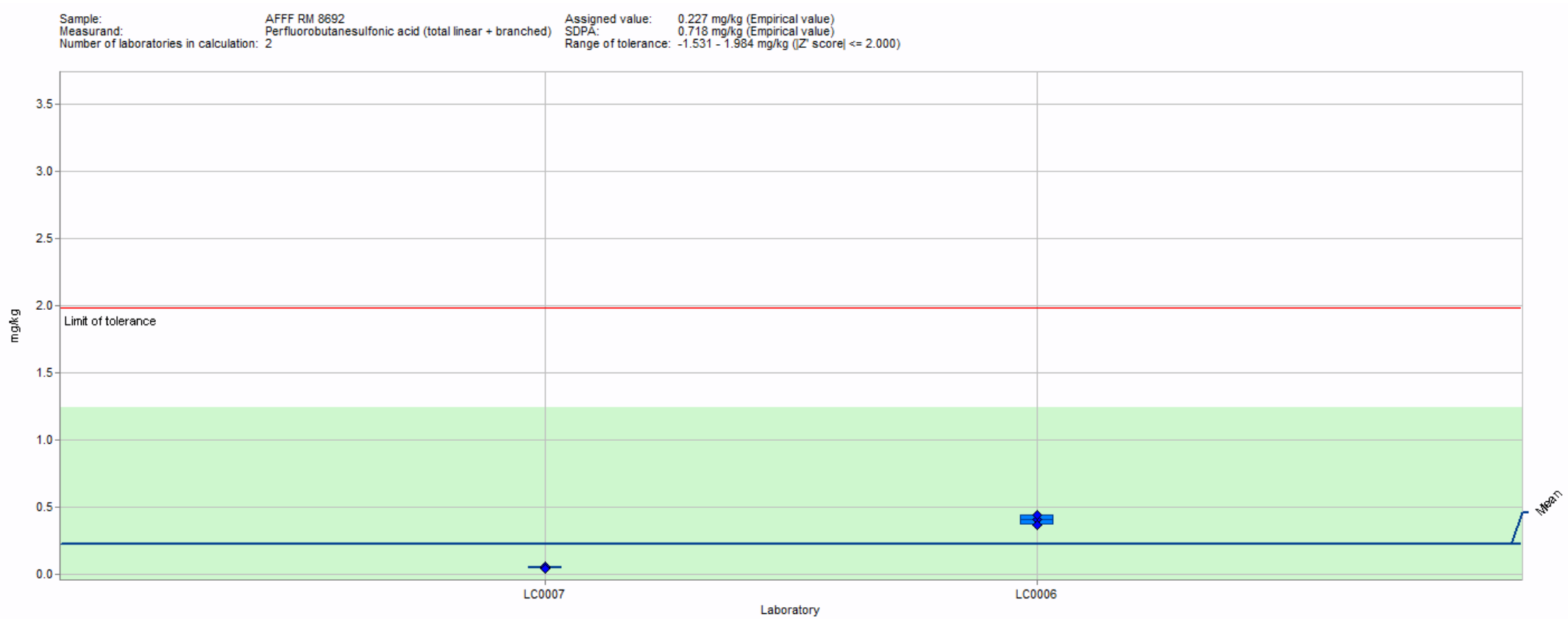

Figure 10-3. PFBS in candidate RM 8692 Per- and Polyfluoroalkyl Substances (PFAS) in Aqueous Film-Forming Foams (AFFF) Formulation III. In this view, individual laboratory data are plotted (diamonds) with the individual laboratory standard deviation (rectangle). The solid blue line represents the consensus mean, and the green shaded region represents the $95 \%$ confidence interval for the consensus mean. The red solid lines represent the consensus range of tolerance, calculated as the values above and below the consensus mean that result in an acceptable $\mathrm{Z}_{\text {comm }}^{\prime}$ score, $\left|\mathrm{Z}_{\text {comm }}^{\prime}\right| \leq 2$. 


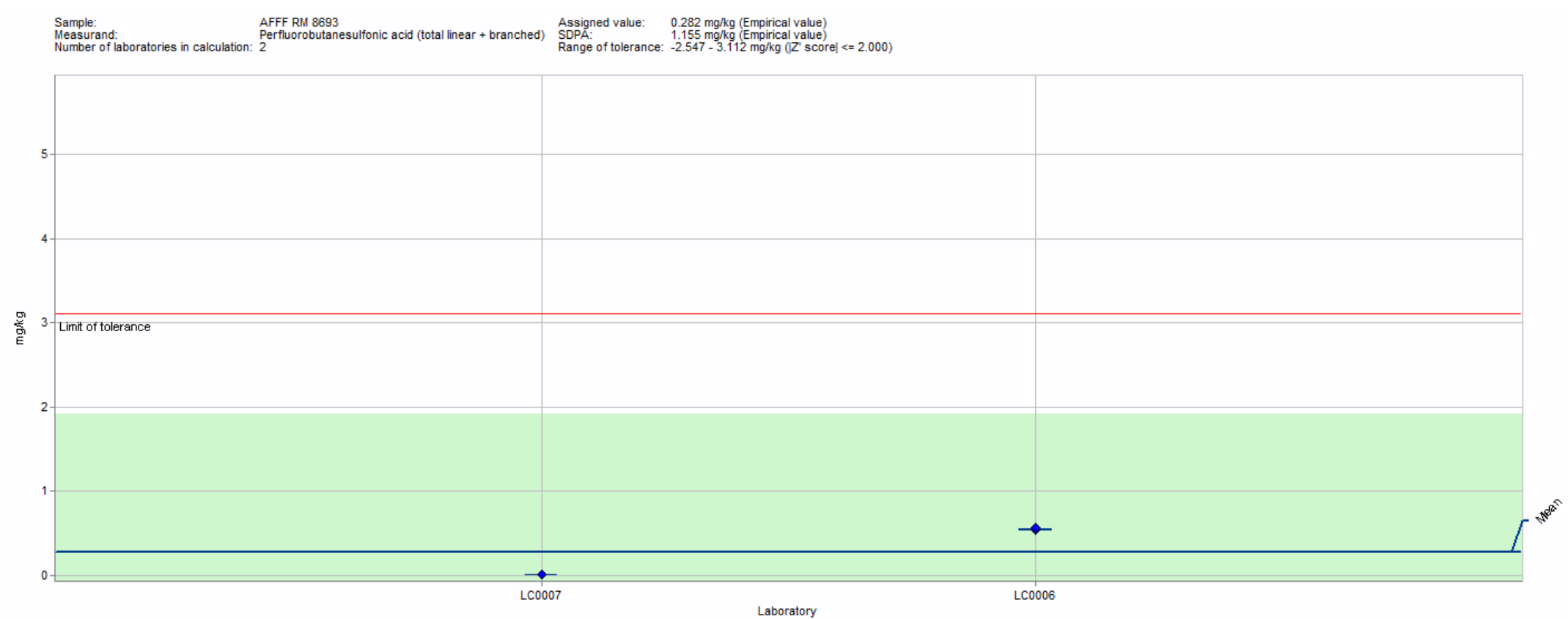

Figure 10-4. PFBS in candidate RM 8693 Per- and Polyfluoroalkyl Substances (PFAS) in Aqueous Film-Forming Foams (AFFF) Formulation IV. In this view, individual laboratory data are plotted (diamonds) with the individual laboratory standard deviation (rectangle). The solid blue line represents the consensus mean, and the green shaded region represents the $95 \%$ confidence interval for the consensus mean. The red solid lines represent the consensus range of tolerance, calculated as the values above and below the consensus mean that result in an acceptable $\mathrm{Z}_{\text {comm }}^{\prime}$ score, $\left|\mathrm{Z}_{\text {comm }}^{\prime}\right| \leq 2$. 


\section{Perfluoropentanesulfonic acid (PFPeS)}

- $\quad$ Six laboratories reported results for PFPeS in candidate RM 8690. Two laboratories reported results for PFPeS in candidate RMs 8691, 8692, and 8693.

- $\quad$ The between-laboratory variability was good for candidate RM 8690 (19\% RSD)

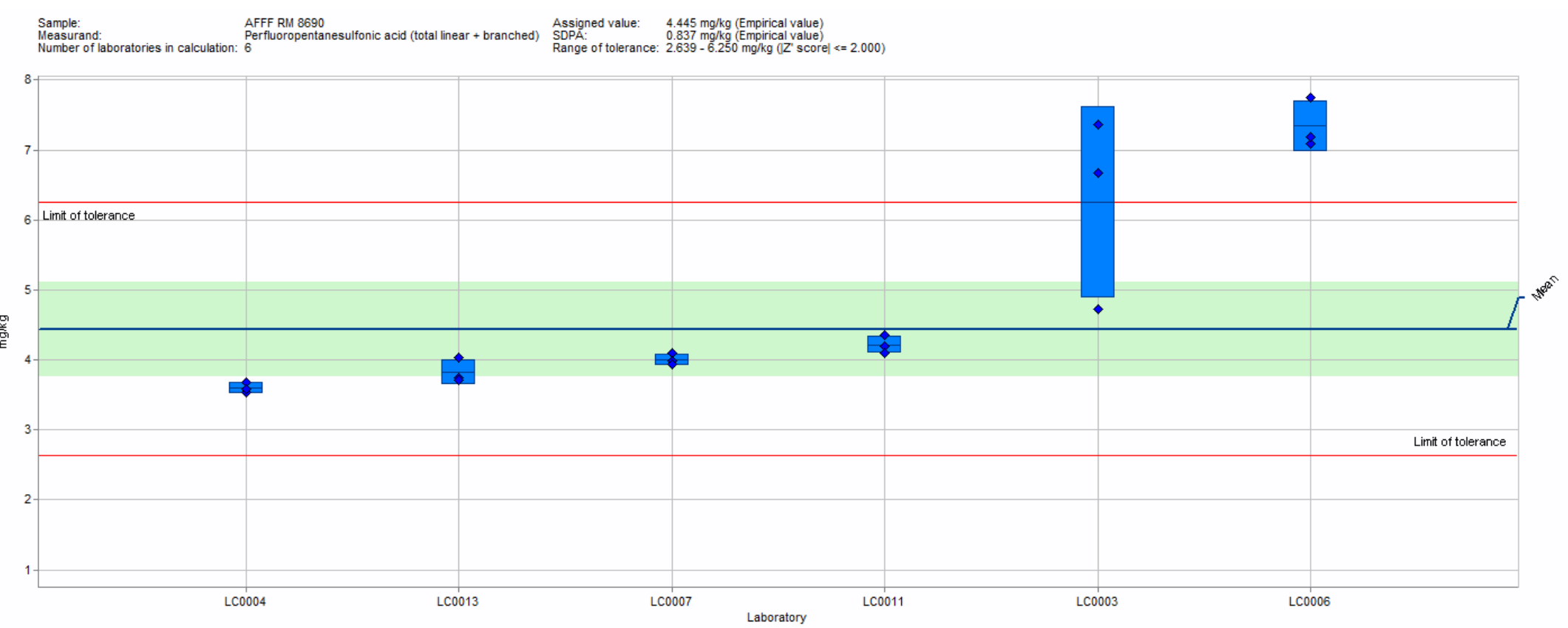

Figure 11. PFPeS in candidate RM 8690 Per- and Polyfluoroalkyl Substances (PFAS) in Aqueous Film-Forming Foams (AFFF) Formulation I. In this view, individual laboratory data are plotted (diamonds) with the individual laboratory standard deviation (rectangle). The solid blue line represents the consensus mean, and the green shaded region represents the $95 \%$ confidence interval for the consensus mean. The red solid lines represent the consensus range of tolerance, calculated as the values above and below the consensus mean that result in an acceptable $\mathrm{Z}_{\text {comm }}^{\prime}$ score, $\left|\mathrm{Z}_{\text {comm }}^{\prime}\right| \leq 2$. 


\section{Perfluorohexanesulfonic acid (PFHxS)}

- Six laboratories reported results for PFHxS in candidate RM 8690. Two laboratories reported results for PFHxS in candidate RMs 8691, 8692, and 8693.

- The between-laboratory variability was poor for candidate RM 8690 (26\% RSD)

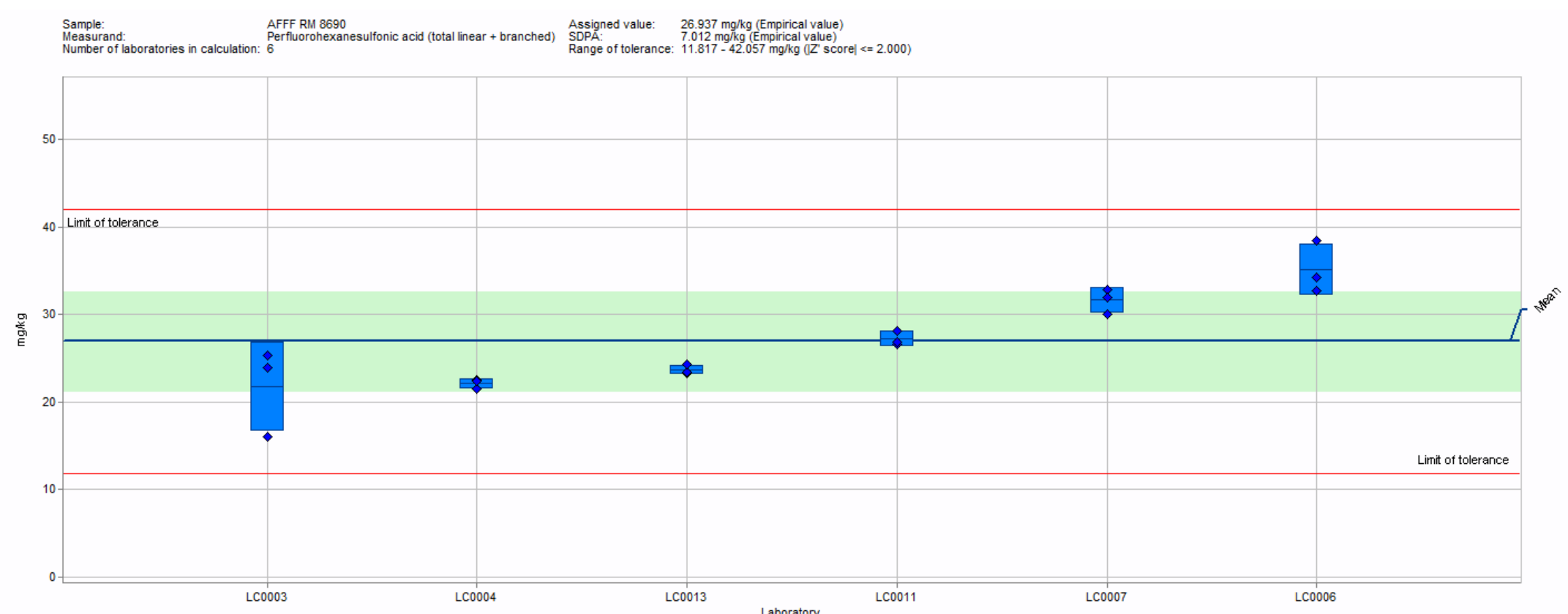

Figure 12. PFHxS in candidate RM 8690 Per- and Polyfluoroalkyl Substances (PFAS) in Aqueous Film-Forming Foams (AFFF) Formulation I. In this view, individual laboratory data are plotted (diamonds) with the individual laboratory standard deviation (rectangle). The solid blue line represents the consensus mean, and the green shaded region represents the $95 \%$ confidence interval for the consensus mean. The red solid lines represent the consensus range of tolerance, calculated as the values above and below the consensus mean that result in an acceptable $\mathrm{Z}_{\text {comm }}^{\prime}$ score, $\left|\mathrm{Z}_{\text {comm }}^{\prime}\right| \leq 2$. 


\section{Perfluoroheptanesulfonic acid (PFHpS)}

- $\quad$ Six laboratories reported results for PFHpS in candidate RM 8690. Three laboratories reported results for PFHpS in candidate RM 8691. Two laboratories reported results for PFHpS in candidate RMs 8692 and 8693.

- $\quad$ The between-laboratory variability was poor for candidate RM 8690 (69 \% RSD)

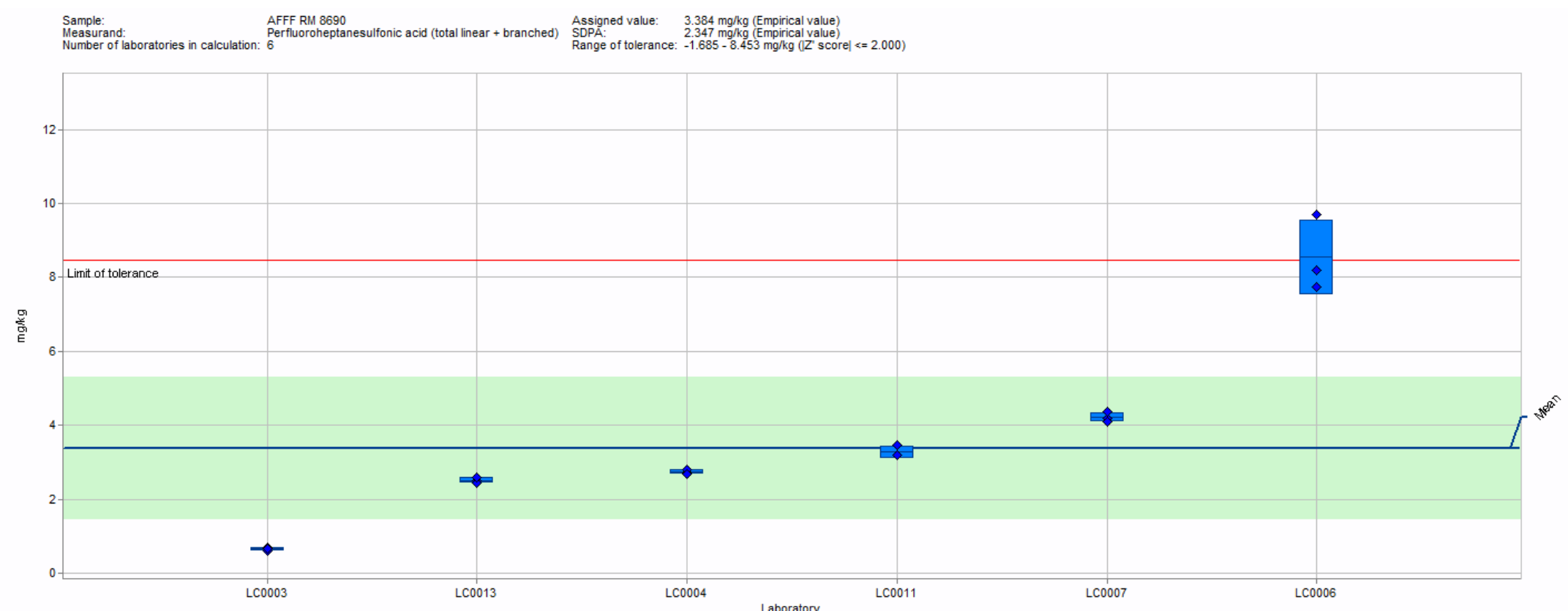

Figure 13. PFHpS in candidate RM 8690 Per- and Polyfluoroalkyl Substances (PFAS) in Aqueous Film-Forming Foams (AFFF) Formulation I. In this view, individual laboratory data are plotted (diamonds) with the individual laboratory standard deviation (rectangle). The solid blue line represents the consensus mean, and the green shaded region represents the $95 \%$ confidence interval for the consensus mean. The red solid lines represent the consensus range of tolerance, calculated as the values above and below the consensus mean that result in an acceptable $\mathrm{Z}_{\text {comm }}^{\prime}$ score, $\left|\mathrm{Z}_{\text {comm }}^{\prime}\right| \leq 2$. 
- $\quad$ Six laboratories reported results for PFOS in candidate RM 8690. Three laboratories reported results for PFOS in candidate RMs 8691, 8692, and 8693.

- The between-laboratory variability was poor for candidate RMs 8690 and 8692 (24\% and $421 \%$ RSD, respectively)

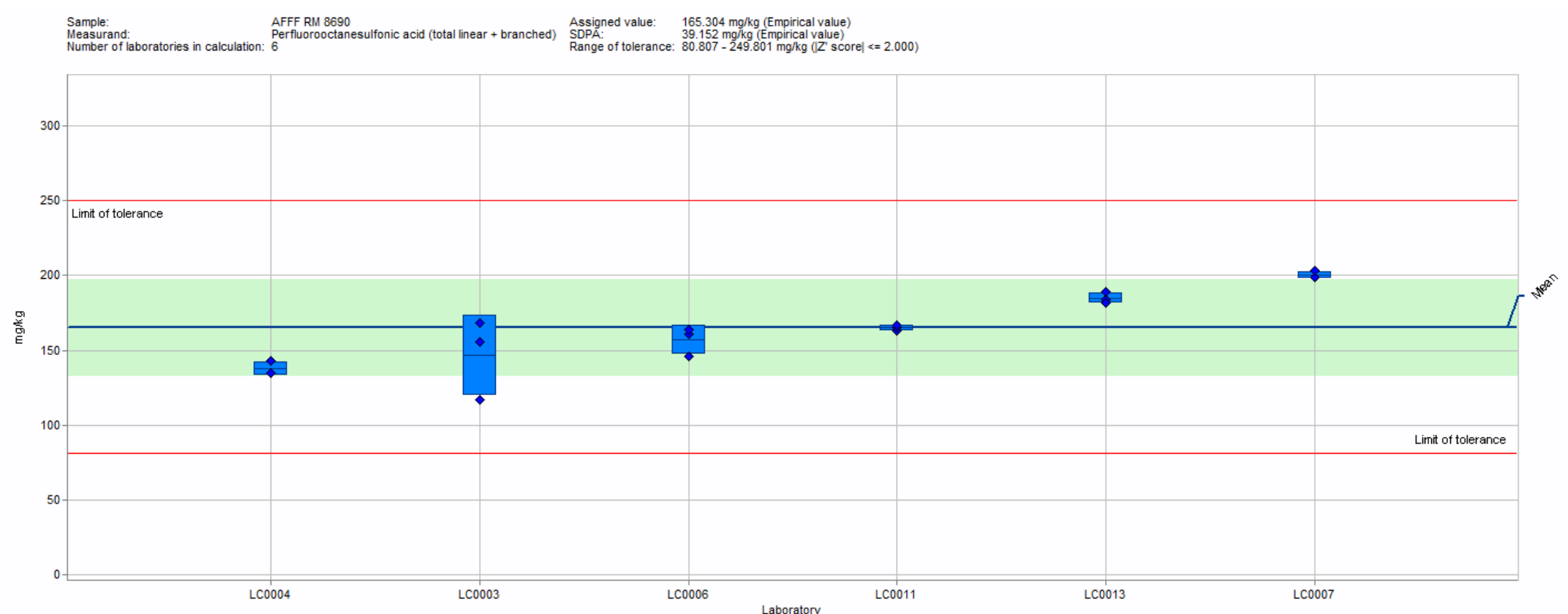

Figure 14-1. PFOS in candidate RM 8690 Per- and Polyfluoroalkyl Substances (PFAS) in Aqueous Film-Forming Foams (AFFF) Formulation I. In this view, individual laboratory data are plotted (diamonds) with the individual laboratory standard deviation (rectangle). The solid blue line represents the consensus mean, and the green shaded region represents the $95 \%$ confidence interval for the consensus mean. The red solid lines represent the consensus range of tolerance, calculated as the values above and below the consensus mean that result in an acceptable $\mathrm{Z}_{\text {comm }}^{\prime}$ score, $\left|\mathrm{Z}_{\text {comm }}^{\prime}\right| \leq 2$. 


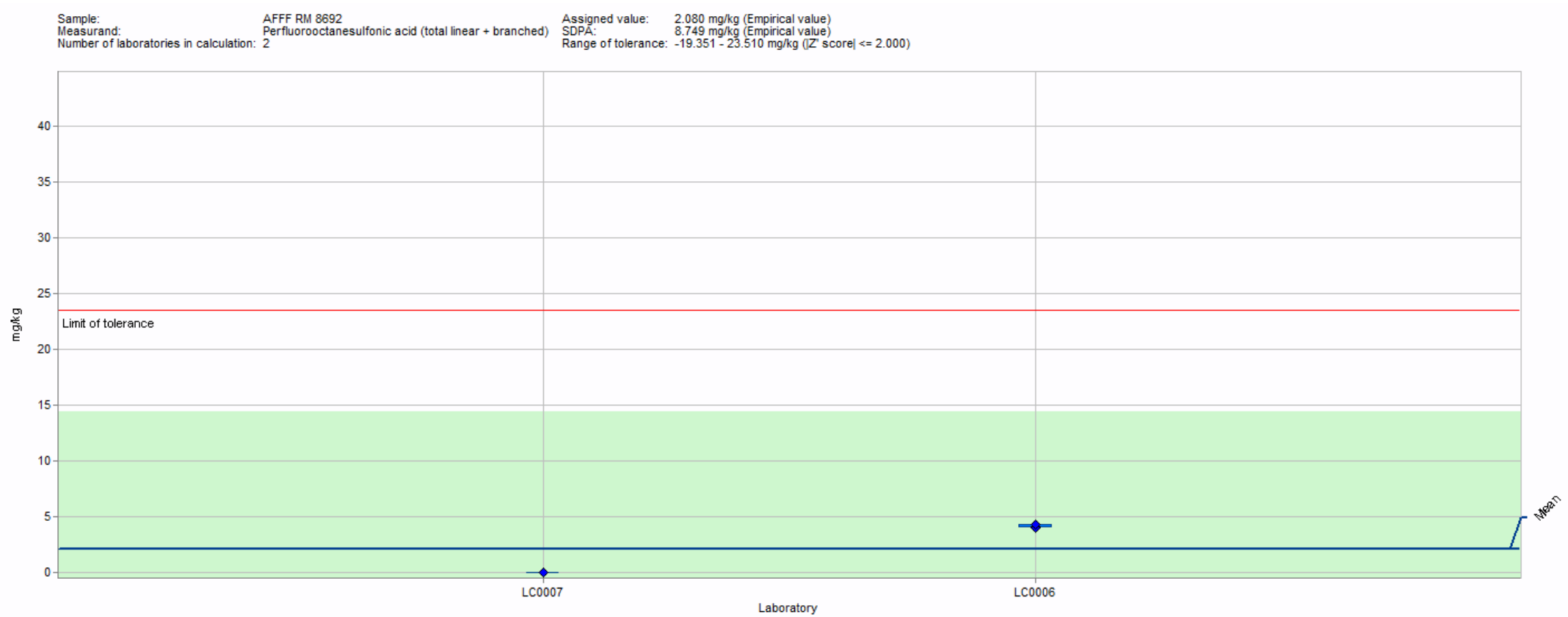

Figure 14-2. PFOS in candidate RM 8692 Per- and Polyfluoroalkyl Substances (PFAS) in Aqueous Film-Forming Foams (AFFF) Formulation III. In this view, individual laboratory data are plotted (diamonds) with the individual laboratory standard deviation (rectangle). The solid blue line represents the consensus mean, and the green shaded region represents the $95 \%$ confidence interval for the consensus mean. The red solid lines represent the consensus range of tolerance, calculated as the values above and below the consensus mean that result in an acceptable $\mathrm{Z}_{\text {comm }}^{\prime}$ score, $\left|\mathrm{Z}_{\text {comm }}^{\prime}\right| \leq 2$. 


\section{Perfluorononanesulfonic acid (PFNS)}

- Three laboratories reported results for PFNS in candidate RMs 8690 and 8692 . Two laboratories reported results for PFNS in candidate RMs 8691 and 8693.

- $\quad$ The between-laboratory variability was poor for candidate RM 8690 (150\% RSD)

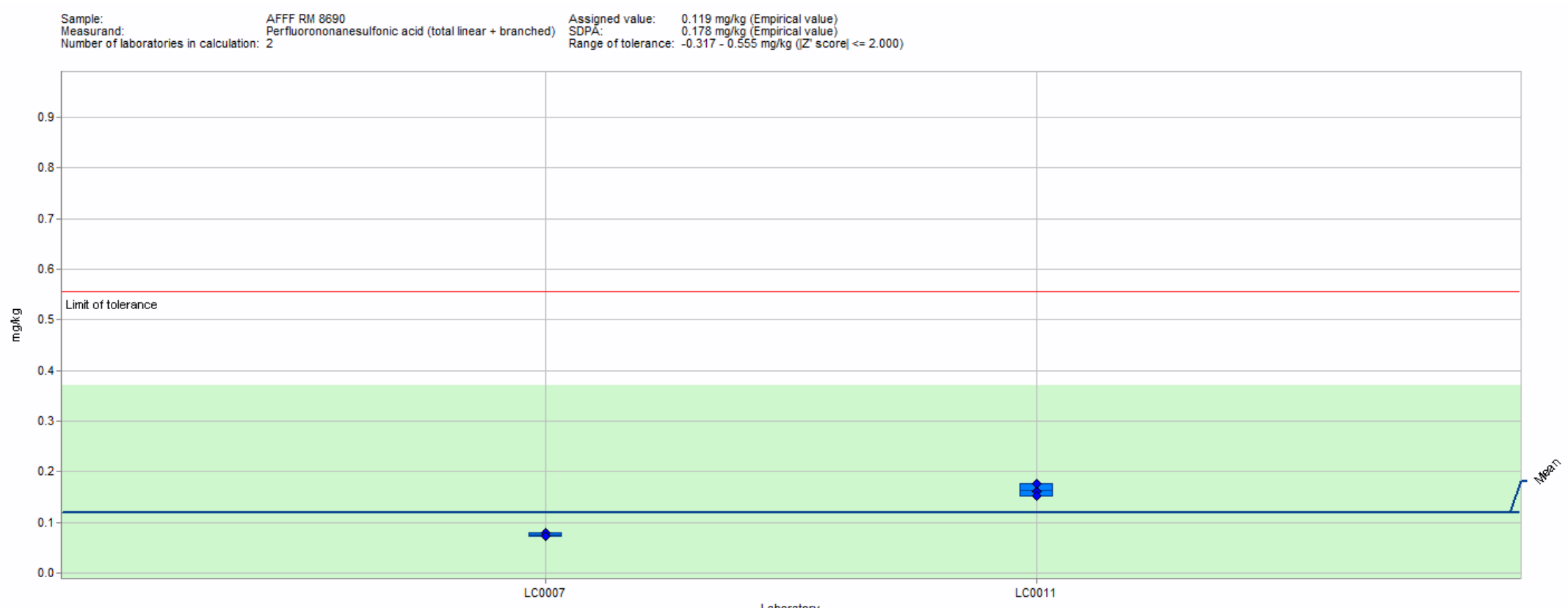

Figure 15. PFNS in candidate RM 8690 Per- and Polyfluoroalkyl Substances (PFAS) in Aqueous Film-Forming Foams (AFFF) Formulation I. In this view, individual laboratory data are plotted (diamonds) with the individual laboratory standard deviation (rectangle). The solid blue line represents the consensus mean, and the green shaded region represents the $95 \%$ confidence interval for the consensus mean. The red solid lines represent the consensus range of tolerance, calculated as the values above and below the consensus mean that result in an acceptable $\mathrm{Z}_{\text {comm }}^{\prime}$ score, $\left|\mathrm{Z}_{\text {comm }}^{\prime}\right| \leq 2$. 
- $\quad$ Four laboratories reported results for PFDS in candidate RM 8690. Two laboratories reported results for PFDS in candidate RMs 8691, 8692, and 8693.

- $\quad$ The between-laboratory variability was poor for candidate RM 8690 (44 \% RSD)

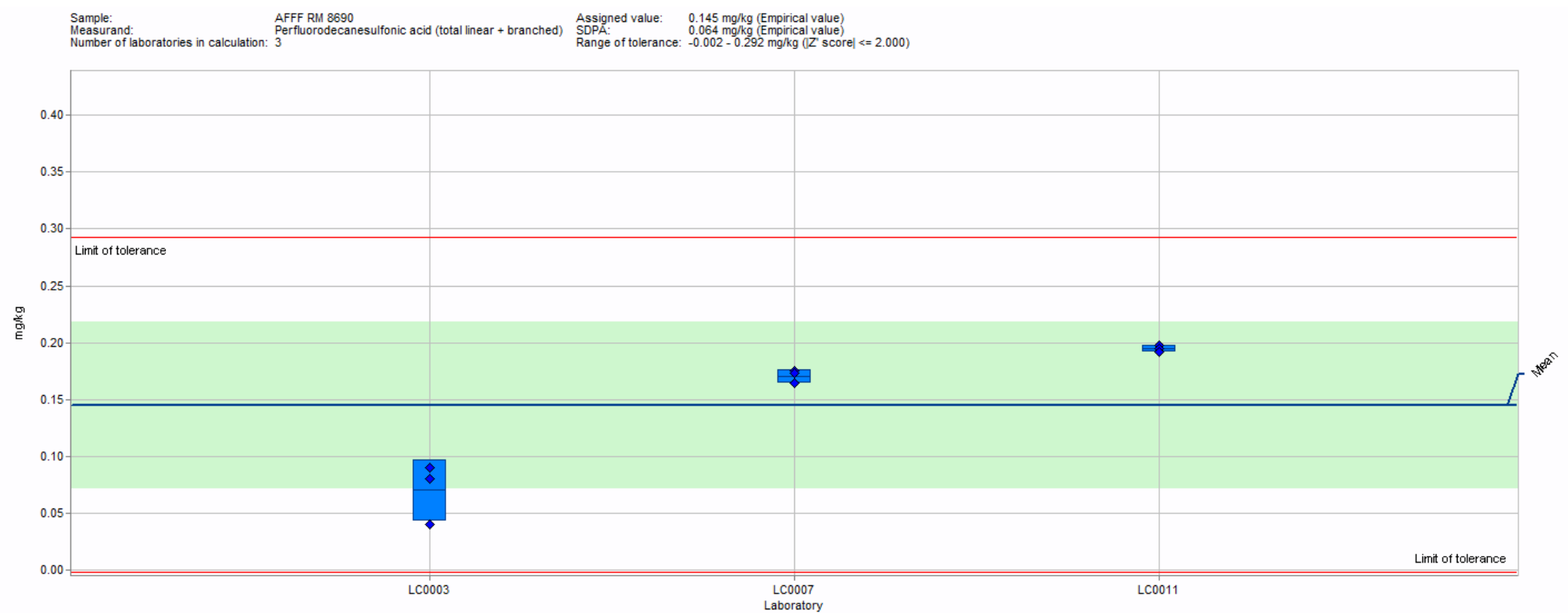

Figure 16. PFDS in candidate RM 8690 Per- and Polyfluoroalkyl Substances (PFAS) in Aqueous Film-Forming Foams (AFFF) Formulation I. In this view, individual laboratory data are plotted (diamonds) with the individual laboratory standard deviation (rectangle). The solid blue line represents the consensus mean, and the green shaded region represents the $95 \%$ confidence interval for the consensus mean. The red solid lines represent the consensus range of tolerance, calculated as the values above and below the consensus mean that result in an acceptable $\mathrm{Z}_{\text {comm }}^{\prime}$ score, $\left|\mathrm{Z}_{\text {comm }}^{\prime}\right| \leq 2$. 
1H,1H, 2H, 2H-Perfluorohexane sulfonic acid (4:2 FTS)

- $\quad$ Six laboratories reported results for 4:2 FTS in candidate RM 8693. Four laboratories reported results for 4:2 FTS in candidate RM 8690. Three laboratories reported results for 4:2 FTS in candidate RM 8691. Two laboratories reported results for 4:2 FTS in candidate RM 8692.

- The between-laboratory variability was poor for candidate RM 8690, 8691, and 8693 (77\%, $344 \%$, and $44 \%$ RSD, respectively).

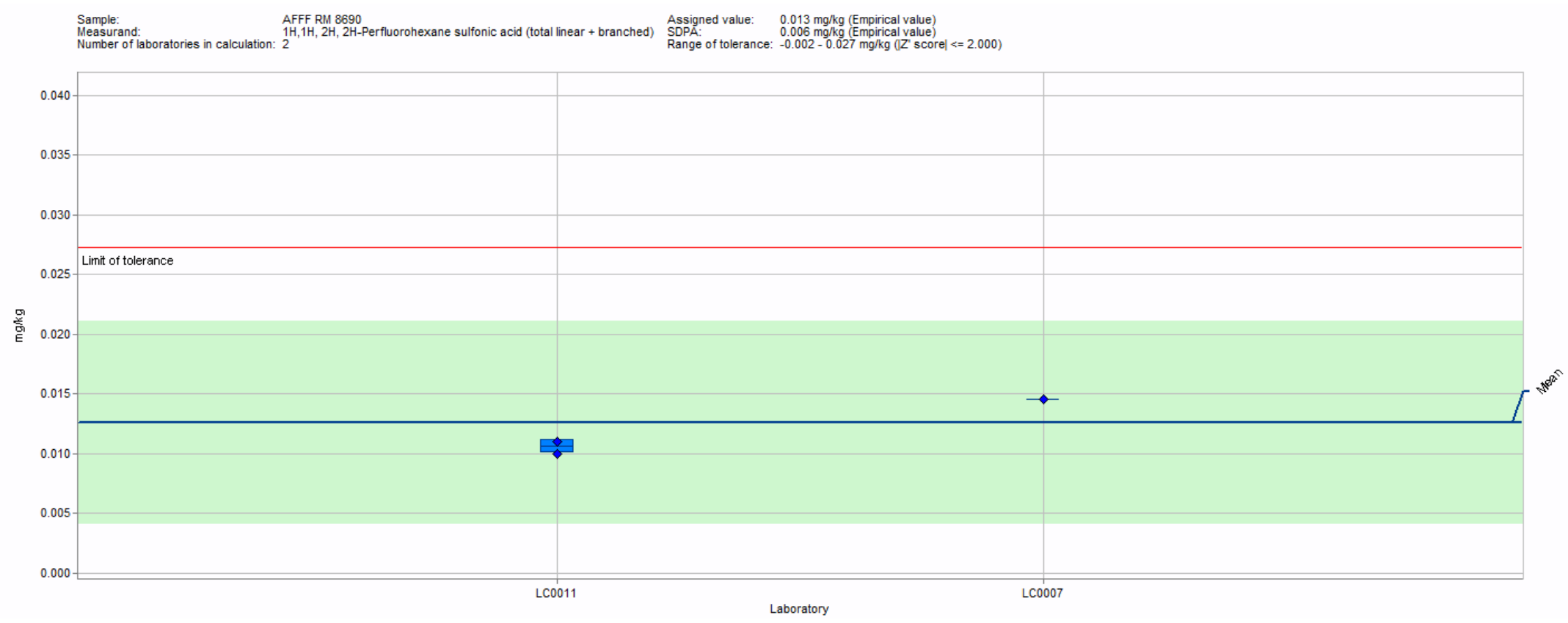

Figure 17-1. 4:2 FTS in candidate RM 8690 Per- and Polyfluoroalkyl Substances (PFAS) in Aqueous Film-Forming Foams (AFFF) Formulation I. In this view, individual laboratory data are plotted (diamonds) with the individual laboratory standard deviation (rectangle). The solid blue line represents the consensus mean, and the green shaded region represents the $95 \%$ confidence interval for the consensus mean. The red solid lines represent the consensus range of tolerance, calculated as the values above and below the consensus mean that result in an acceptable $\mathrm{Z}_{\text {comm }}^{\prime}$ score, $\left|\mathrm{Z}_{\text {comm }}^{\prime}\right| \leq 2$. 


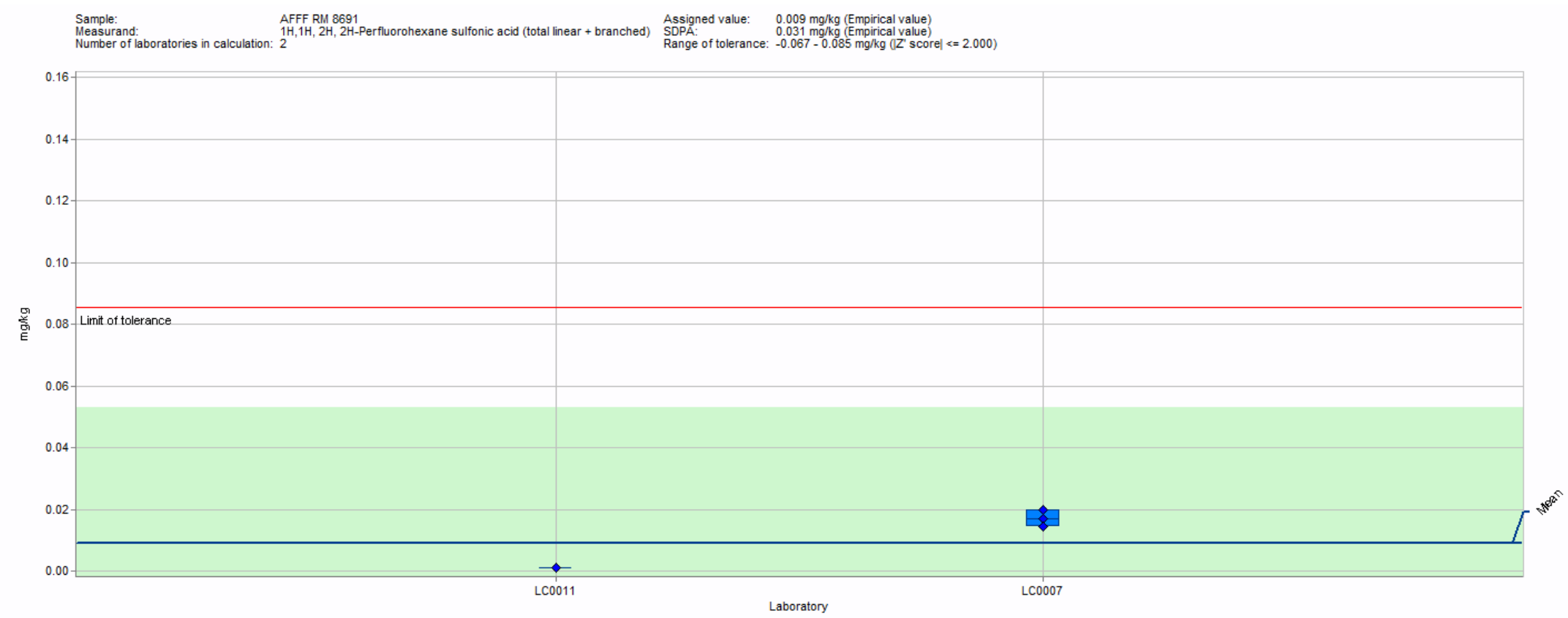

Figure 17-2. 4:2 FTS in candidate RM 8691 Per- and Polyfluoroalkyl Substances (PFAS) in Aqueous Film-Forming Foams (AFFF) Formulation II. In this view, individual laboratory data are plotted (diamonds) with the individual laboratory standard deviation (rectangle). The solid blue line represents the consensus mean, and the green shaded region represents the $95 \%$ confidence interval for the consensus mean. The red solid lines represent the consensus range of tolerance, calculated as the values above and below the consensus mean that result in an acceptable $\mathrm{Z}_{\text {comm }}^{\prime}$ score, $\left|\mathrm{Z}_{\text {comm }}^{\prime}\right| \leq 2$. 


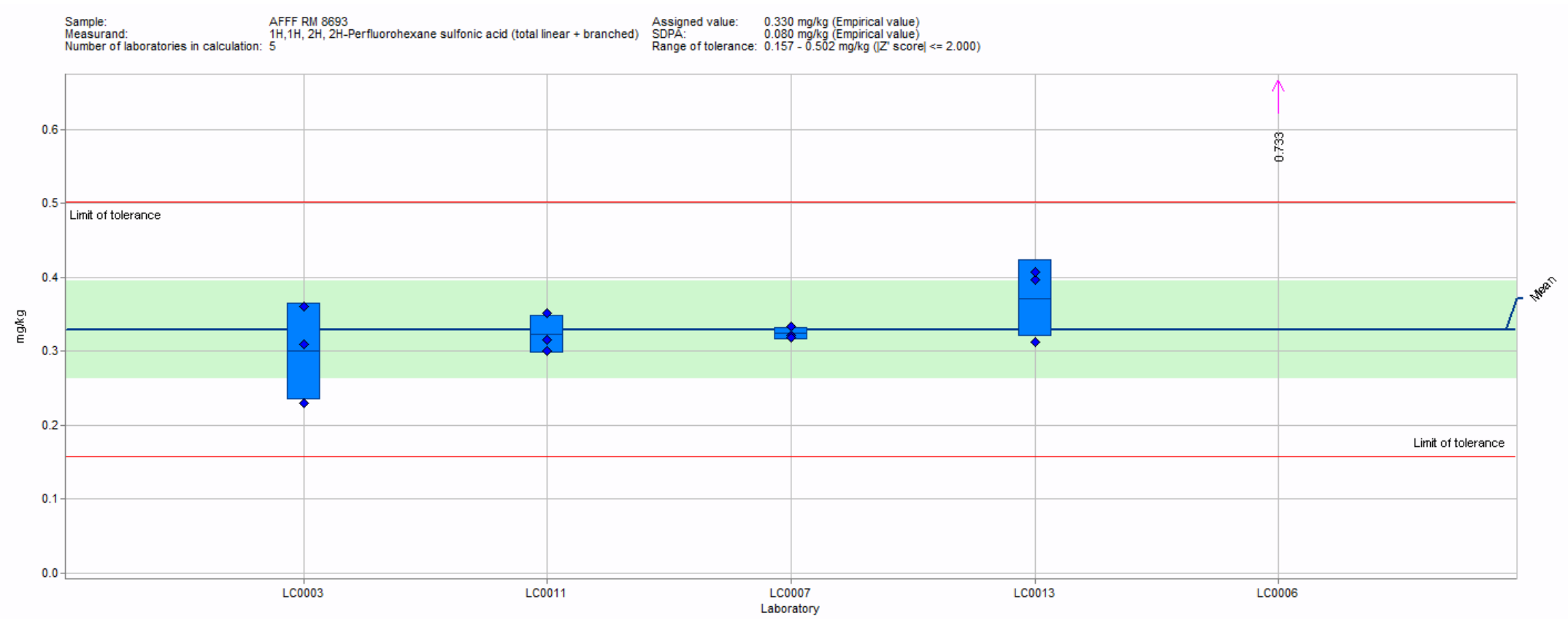

Figure 17-3. 4:2 FTS in candidate RM 8693 Per- and Polyfluoroalkyl Substances (PFAS) in Aqueous Film-Forming Foams (AFFF) Formulation IV. In this view, individual laboratory data are plotted (diamonds) with the individual laboratory standard deviation (rectangle). The solid blue line represents the consensus mean, and the green shaded region represents the $95 \%$ confidence interval for the consensus mean. The red solid lines represent the consensus range of tolerance, calculated as the values above and below the consensus mean that result in an acceptable $\mathrm{Z}_{\text {comm }}^{\prime}$ score, $\left|\mathrm{Z}_{\text {comm }}^{\prime}\right| \leq 2$. 
$1 H, 1 H, 2 H, 2 H$-Perfluorooctane sulfonic acid (6:2 FTS)

- Six laboratories reported results for 6:2 FTS in candidate RMs 8690 and 8693. Five laboratories reported results for 6:2 FTS in candidate RMs 8691 and 8692.

- $\quad$ The between-laboratory variability was poor for candidate RM 8690, 8691, 8692 and 8693 (65 \%, 24 \%, $49 \%$, and $26 \%$ RSD, respectively).

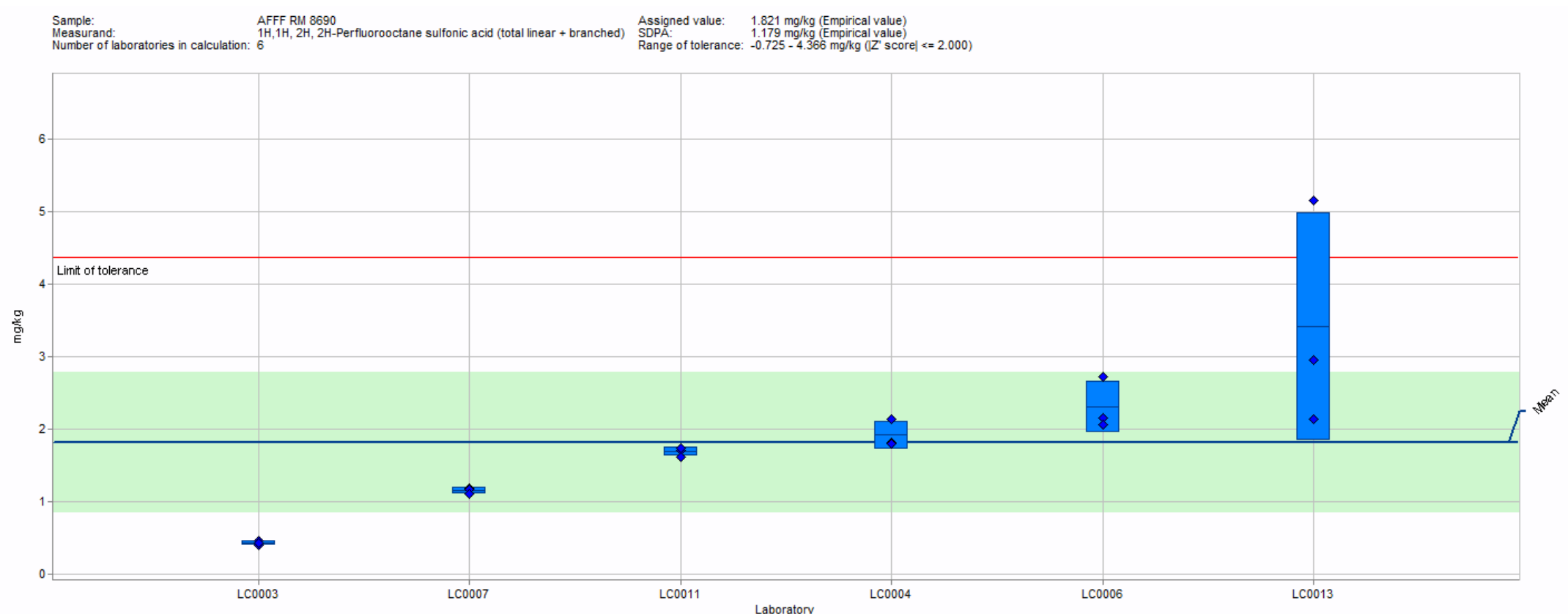

Figure 18-1. 6:2 FTS in candidate RM 8690 Per- and Polyfluoroalkyl Substances (PFAS) in Aqueous Film-Forming Foams (AFFF) Formulation I. In this view, individual laboratory data are plotted (diamonds) with the individual laboratory standard deviation (rectangle). The solid blue line represents the consensus mean, and the green shaded region represents the $95 \%$ confidence interval for the consensus mean. The red solid lines represent the consensus range of tolerance, calculated as the values above and below the consensus mean that result in an acceptable $\mathrm{Z}_{\text {comm }}^{\prime}$ score, $\left|\mathrm{Z}_{\text {comm }}^{\prime}\right| \leq 2$. 


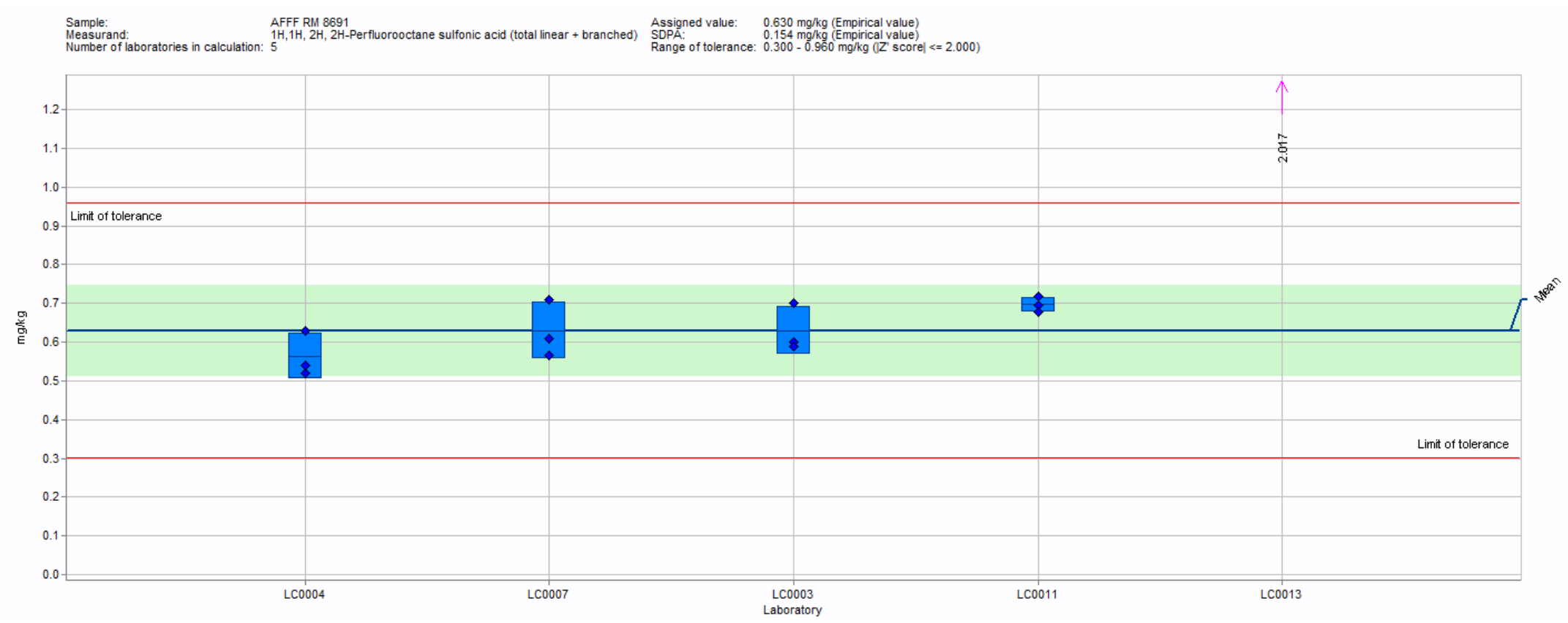

Figure 18-2. 6:2 FTS in candidate RM 8691 Per- and Polyfluoroalkyl Substances (PFAS) in Aqueous Film-Forming Foams (AFFF) Formulation II. In this view, individual laboratory data are plotted (diamonds) with the individual laboratory standard deviation (rectangle). The solid blue line represents the consensus mean, and the green shaded region represents the $95 \%$ confidence interval for the consensus mean. The red solid lines represent the consensus range of tolerance, calculated as the values above and below the consensus mean that result in an acceptable $\mathrm{Z}_{\text {comm }}^{\prime}$ score, $\left|\mathrm{Z}_{\text {comm }}^{\prime}\right| \leq 2$. 


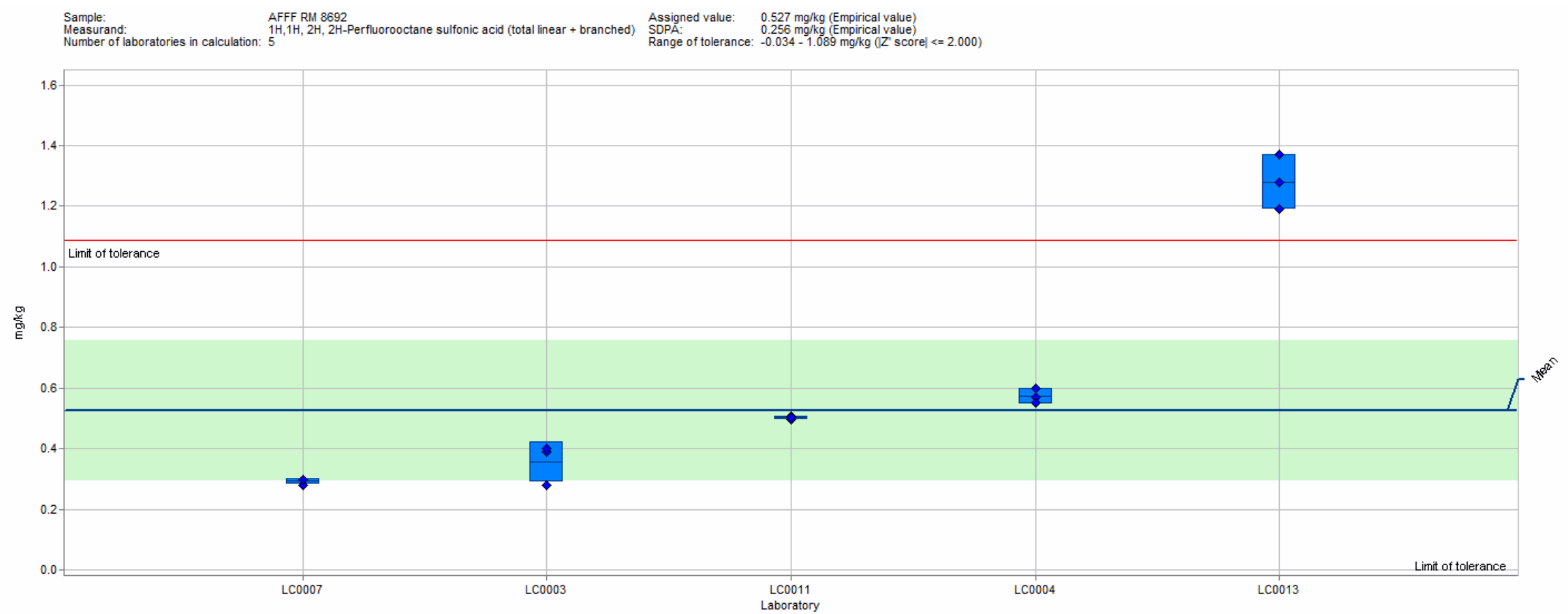

Figure 18-3. 6:2 FTS in candidate RM 8692 Per- and Polyfluoroalkyl Substances (PFAS) in Aqueous Film-Forming Foams (AFFF) Formulation III. In this view, individual laboratory data are plotted (diamonds) with the individual laboratory standard deviation (rectangle). The solid blue line represents the consensus mean, and the green shaded region represents the $95 \%$ confidence interval for the consensus mean. The red solid lines represent the consensus range of tolerance, calculated as the values above and below the consensus mean that result in an acceptable $\mathrm{Z}_{\text {comm }}^{\prime}$ score, $\left|\mathrm{Z}_{\text {comm }}^{\prime}\right| \leq 2$. 


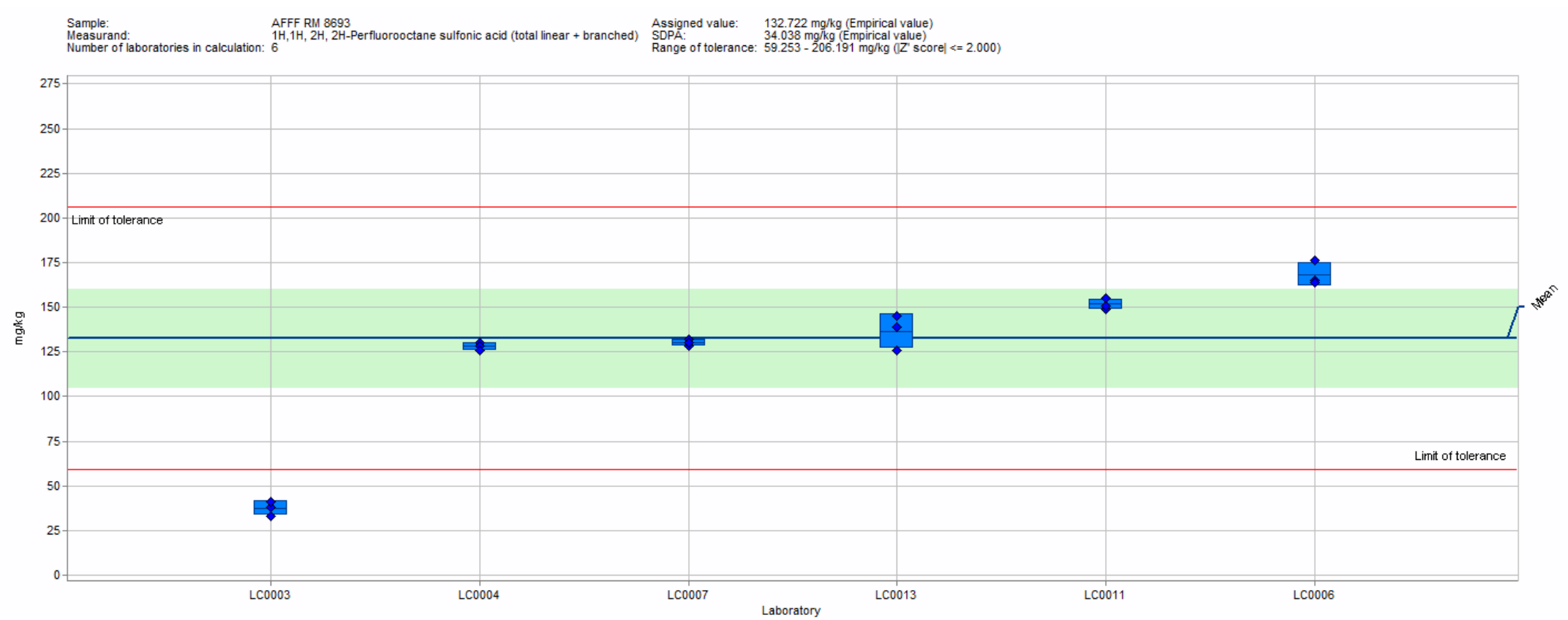

Figure 18-4. 6:2 FTS in candidate RM 8693 Per- and Polyfluoroalkyl Substances (PFAS) in Aqueous Film-Forming Foams (AFFF) Formulation IV. In this view, individual laboratory data are plotted (diamonds) with the individual laboratory standard deviation (rectangle). The solid blue line represents the consensus mean, and the green shaded region represents the $95 \%$ confidence interval for the consensus mean. The red solid lines represent the consensus range of tolerance, calculated as the values above and below the consensus mean that result in an acceptable $\mathrm{Z}_{\text {comm }}^{\prime}$ score, $\left|\mathrm{Z}_{\text {comm }}^{\prime}\right| \leq 2$. 
- Four laboratories reported results for 8:2 FTS in candidate RMs 8690, 8691, and 8693. Three laboratories reported results for 8:2 FTS in candidate RM 8692.

- The between-laboratory variability was poor for candidate RM 8690, 8691, and $8693(80 \%$, $113 \%$, and $517 \%$ RSD, respectively).

- One laboratory reported results of over $250 \mathrm{mg} / \mathrm{kg}$ in candidate RM 8693. Since this was substantially higher compared to the other reported values (around $0.112 \mathrm{mg} / \mathrm{kg}$ ), we went back to the material and found a chemical interferant that when not fully separated from 8:2 FTS, gave a substantially higher reported value. This chemical interferant is not 8:2 FTS, but does have a similar retention time and primary MS/MS transition.

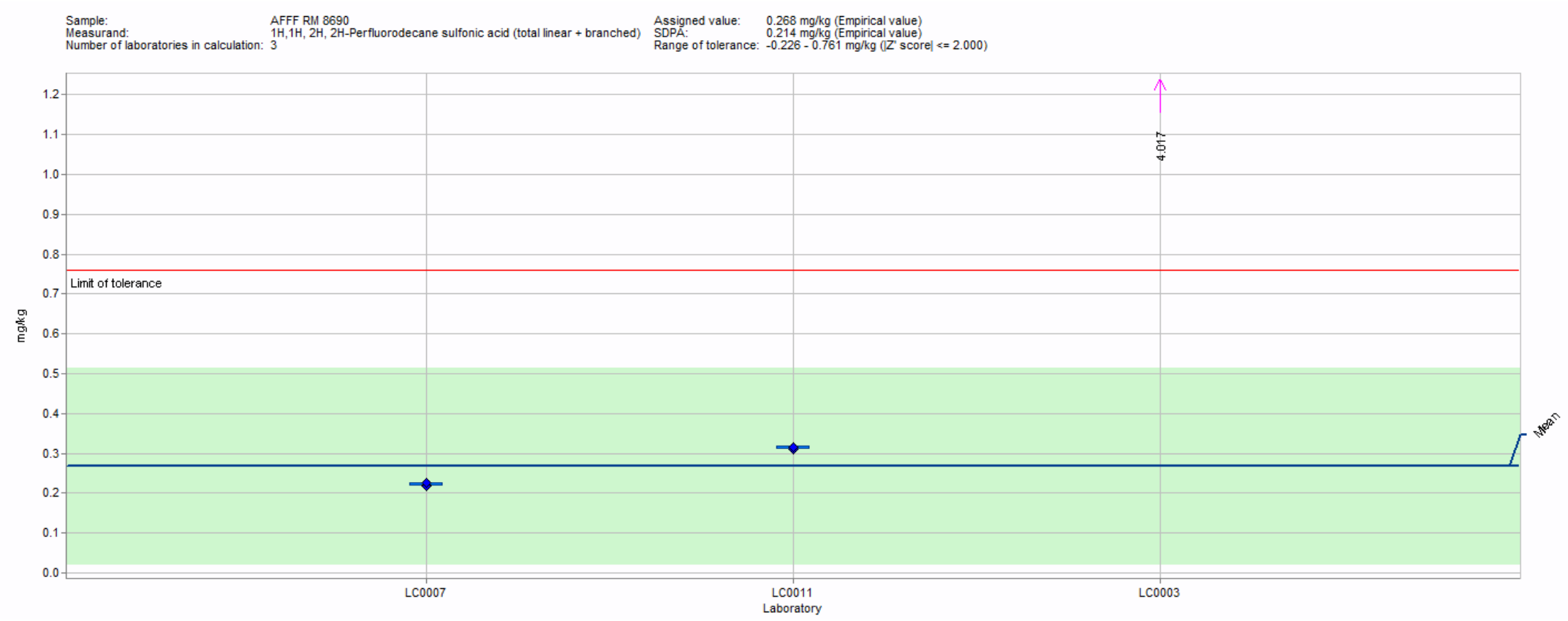

Figure 19-1. 8:2 FTS in candidate RM 8690 Per- and Polyfluoroalkyl Substances (PFAS) in Aqueous Film-Forming Foams (AFFF) Formulation I. In this view, individual laboratory data are plotted (diamonds) with the individual laboratory standard deviation (rectangle). The solid blue line represents the consensus mean, and the green shaded region represents the $95 \%$ confidence interval for the consensus mean. The red solid lines represent the consensus range of tolerance, calculated as the values above and below the consensus mean that result in an acceptable $\mathrm{Z}_{\text {comm }}^{\prime}$ score, $\left|\mathrm{Z}_{\text {comm }}^{\prime}\right| \leq 2$. 


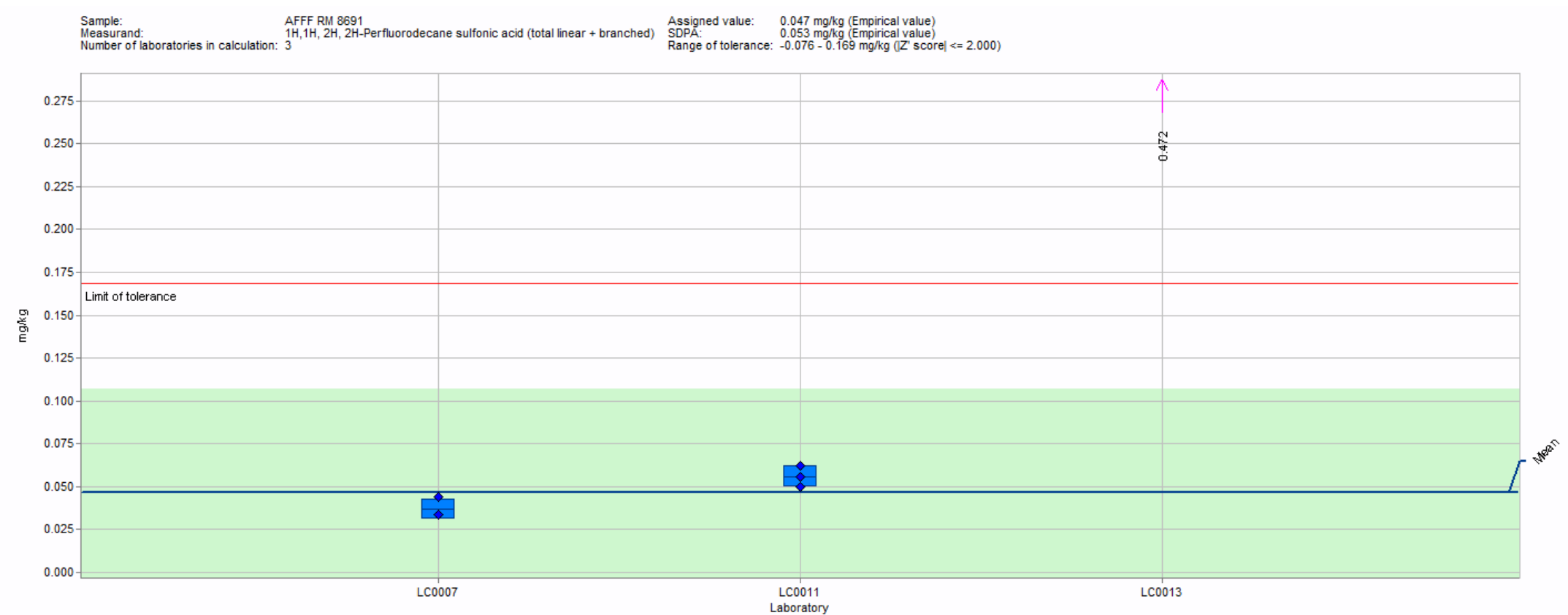

Figure 19-2. 8:2 FTS in candidate RM 8691 Per- and Polyfluoroalkyl Substances (PFAS) in Aqueous Film-Forming Foams (AFFF) Formulation II. In this view, individual laboratory data are plotted (diamonds) with the individual laboratory standard deviation (rectangle). The solid blue line represents the consensus mean, and the green shaded region represents the $95 \%$ confidence interval for the consensus mean. The red solid lines represent the consensus range of tolerance, calculated as the values above and below the consensus mean that result in an acceptable $\mathrm{Z}_{\text {comm }}^{\prime}$ score, $\left|\mathrm{Z}_{\text {comm }}^{\prime}\right| \leq 2$. 


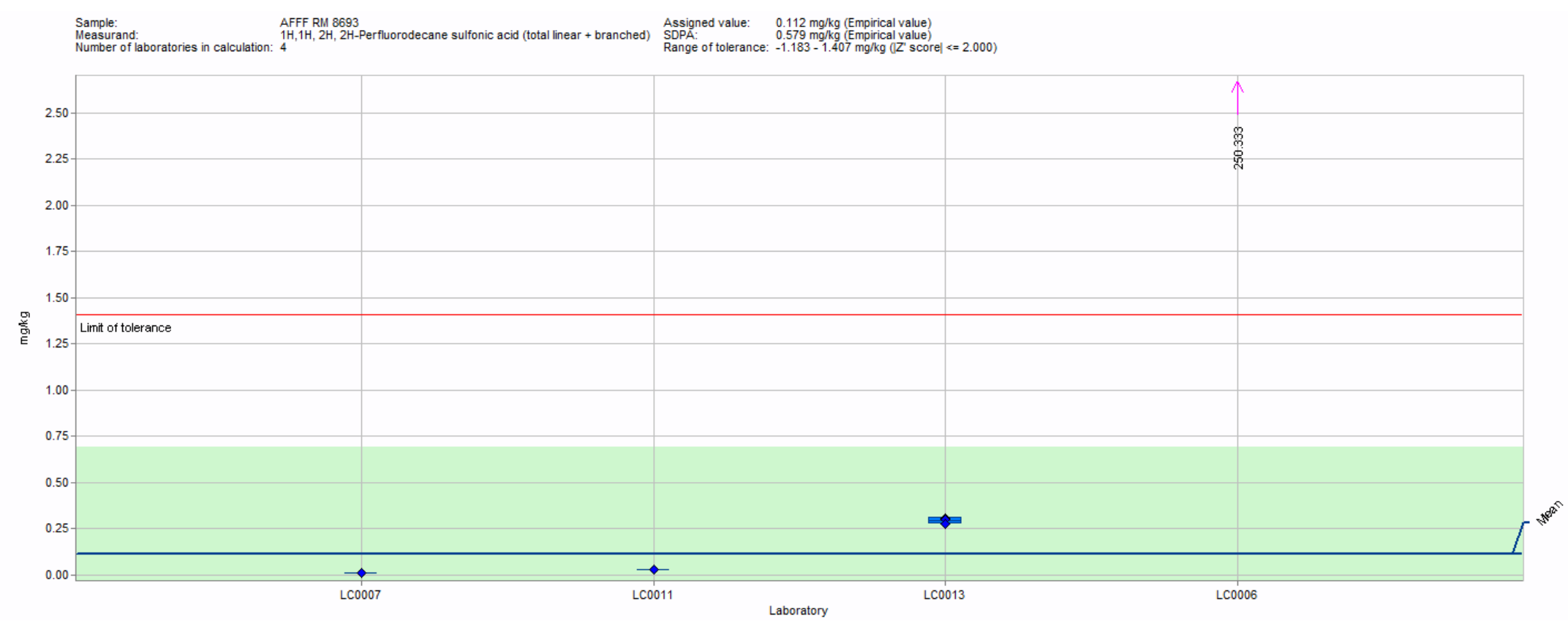

Figure 19-3. 8:2 FTS in candidate RM 8693 Per- and Polyfluoroalkyl Substances (PFAS) in Aqueous Film-Forming Foams (AFFF) Formulation IV. In this view, individual laboratory data are plotted (diamonds) with the individual laboratory standard deviation (rectangle). The solid blue line represents the consensus mean, and the green shaded region represents the $95 \%$ confidence interval for the consensus mean. The red solid lines represent the consensus range of tolerance, calculated as the values above and below the consensus mean that result in an acceptable $\mathrm{Z}_{\text {comm }}^{\prime}$ score, $\left|\mathrm{Z}_{\text {comm }}^{\prime}\right| \leq 2$. 


\section{Conclusions}

There are technical recommendations based on feedback from some of the participants in this study.

1) Laboratories reported foaming of the candidate materials which made it difficult to subsample from the vials. It is recommended that the candidate RMs be diluted (approximately 1:10 v:v) to make material handling easier and reduce the chance of foaming.

2) Given the large \% RSD values for most analytes, measuring PFAS in AFFFs is clearly a challenging measurement. AFFF RMs will be useful for to validate future measurements of PFAS in AFFF or other commercial formulations.

3) The use of matrix RMs for method validation and quality assurance of the measurement process is recommended. 


\section{References}

${ }^{1}$ Reiner, J.L.; Phinney, K.W.; Keller, J.M. Determination of perfluorinated compounds in human plasma Standard Reference Material. Anal. Bioanal Chem. 2011. 9, 2899-2907.

${ }^{2}$ Reiner, J.L.; O’Connell, S.G.; Butt, C.; Mabury, S.; Small, J.; De Silva, A.O.; Muir, D.; Delinsky, A.; Strynar, M.J.; Lindstrom, A.B.; Reagen, W.; Malinsky, M.; Schäfer, S.; Kwadijk, C.; Schantz, M.M.; Keller, J.M. Determination of Perfluorinated Alkyl Acid Concentrations in Biological Standard Reference Materials. Anal Bioanal Chem. 2012. 9, 2683-2692.

${ }^{3}$ Reiner, J.L.; Blaine, A.C.; Higgins, C. P.; Huset, C.; Jenkins, T.M.; Kwadijk, C.; Lange, C.; Muir, D.C.G.; Reagen, W.; Rich, C.; Small, J.M.; Strynar, M.J.; Washington, J.W.; Yoo, H.; Keller, J.M. Polyfluorinated Substances in Abiotic Standard Reference Materials. Anal Bioanal Chem. 2015. 407, 2975-2983.

${ }^{4}$ ISO 13528:2015(E), Statistical methods for use in proficiency testing by interlaboratory comparisons, pp. 53-54. 